\title{
THE INVISIBLES: A DETECTION ALGORITHM TO TRACE THE FAINTEST MILKY WAY SATELLITES
}

\author{
S. M. WALSH ${ }^{1}$, B. WILLMAN ${ }^{2,3}$, AND H. JERJEN ${ }^{1}$ \\ ${ }^{1}$ Research School of Astronomy and Astrophysics, Australian National University, Cotter Road, Weston, ACT 2611, Australia; swalsh@mso.anu.edu.au \\ ${ }^{2}$ Clay Fellow, Harvard-Smithsonian Center for Astrophysics, 60 Garden Street, Cambridge, MA 02138, USA \\ ${ }^{3}$ Haverford College, 371 Lancaster Ave, Haverford PA 19041, USA \\ Received 2008 July 19; accepted 2008 October 21; published 2008 December 19
}

\begin{abstract}
A specialized data-mining algorithm has been developed using wide-field photometry catalogs, enabling systematic and efficient searches for resolved, extremely low surface brightness satellite galaxies in the halo of the Milky Way (MW). Tested and calibrated with the Sloan Digital Sky Survey Data Release 6 (SDSS-DR6) we recover all 15 MW satellites recently detected in SDSS, six known MW/Local Group dSphs in the SDSS footprint, and 19 previously known globular and open clusters. In addition, 30 point-source overdensities have been found that correspond to no cataloged objects. The detection efficiencies of the algorithm have been carefully quantified by simulating more than three million model satellites embedded in star fields typical of those observed in SDSS, covering a wide range of parameters including galaxy distance, scale length, luminosity, and Galactic latitude. We present several parameterizations of these detection limits to facilitate comparison between the observed MW satellite population and predictions. We find that all known satellites would be detected with $>90 \%$ efficiency over all latitudes spanned by DR6 and that the MW satellite census within DR6 is complete to a magnitude limit of $M_{V} \approx-6.5$ and a distance of $300 \mathrm{kpc}$. Assuming all existing MW satellites contain an appreciable old stellar population and have sizes and luminosities comparable with currently known companions, we predict lower and upper limit totals of 52 and $340 \mathrm{MW}$ dwarf satellites within $\sim 260 \mathrm{kpc}$ if they are uniformly distributed across the sky. This result implies that many MW satellites still remain undetected. Identifying and studying these elusive satellites in future survey data will be fundamental to test the dark matter distribution on kpc scales.
\end{abstract}

Key words: dark matter - galaxies: dwarf - Local Group

Online-only material: color figures

\section{INTRODUCTION}

The dwarf galaxy population of the Milky Way (MW) provides invaluable insight into galaxy formation and evolution. Their resolved stars reveal formation histories and enable precise measurements of their structural parameters, ages, and metallicities. These histories of individual, nearby systems provide a unique approach to studying the universe across the cosmic ages. Dwarf galaxies are also the most numerous type of galaxy in the universe (González et al. 2006) and are thought to be the building blocks of larger galaxies. Owing to their low masses, their properties may be strongly influenced by ionizing radiation in the early universe (Bullock et al. 2000; Somerville 2002) and by the energy released by supernovae (Dekel \& Silk 1986; Mori et al. 2002). The impacts of both of these are weak links in our understanding of structure formation. Finding and studying nearby dwarfs of the lowest masses and luminosities is thus an essential component to understanding galaxy formation on all scales.

The MW dwarf galaxy population is also at present the most direct tracer of the abundance, mass spectrum, characteristic size, and spatial distribution of dark matter (DM) on subgalactic scales. Standard $\Lambda$ cold dark matter $(\Lambda \mathrm{CDM})$ simulations of MW-size DM halos predict many more DM subhalos than are observed as dwarf galaxies (Klypin et al. 1999; Moore et al. 1999). The recent "Via Lactea" simulation contains $2000 \mathrm{DM}$ subhalos within $289 \mathrm{kpc}$ of the simulated primary galaxy (Diemand et al. 2007) which have no observed optically luminous counterparts. This discrepancy leads to the questions of how and in what mass regime baryons disappear from DM clumps. Studies of the spatial distributions of MW and
M31 dwarf galaxy companions have also highlighted possible discrepancies between $\Lambda$ CDM theory and observations (Kroupa et al. 2005; Kang et al. 2005; Metz et al. 2007).

The most obvious reason for these apparent discrepancies in the number and spatial distributions of dwarf galaxies is substantial incompleteness, as the MW halo has not yet been uniformly searched for dwarf galaxy companions to low enough luminosities and star densities, in particular close to the Galactic plane where foreground contamination is severe. For example, Willman et al. (2004) compared the spatial distribution of MW satellites with that of M31's population, as well as that of a simulated DM halo, and concluded that some dwarfs may have been missed at low Galactic latitudes and that the total number of MW satellites with properties similar to the known objects could be as many as triple the known population.

The viability of this solution, at least in part, has been underscored by the recent discoveries of 14 new Galactic companions from the photometric data of the Sloan Digital Sky Survey (SDSS). These objects all appear to be dominated by old ( $\gtrsim 10 \mathrm{Gyr}$ ) stellar populations, with the exception of Leo T (de Jong et al. 2008). Nine of these companions were immediately identified as dwarf spheroidal $(\mathrm{dSph})$ galaxies: Ursa Major, Canes Venatici, Boötes, Ursa Major II, Canes Venatici II, Hercules, Leo IV, Coma Berenices, Leo T, and Leo V (Willman et al. 2005b; Zucker et al. 2006a; Belokurov et al. 2006b; Zucker et al. 2006b; Belokurov et al. 2007; Irwin et al. 2007; Belokurov et al. 2008). Spectroscopic follow-up has confirmed that they all are highly DM-dominated dwarf galaxies (Simon \& Geha 2007; Martin et al. 2007). Willman 1, Segue 1, and Boötes II (Willman et al. 2005a; Belokurov et al. 2007; Walsh et al. 2007a) occupy a region of size-luminosity space 
not previously known to be inhabited by old stellar populations, at the intersection of MW dSphs and globular clusters. Spectroscopic studies (Martin et al. 2007) showed that Willman 1 may be resident inside a DM subhalo with a mass-to-light ratio of $\sim 470$. If these ambiguous objects are gravitationally bound, then tidal arguments also favor them being DM dominated (Walsh et al. 2007). The remaining two objects discovered in SDSS, Koposov 1 and 2 (Koposov et al. 2007), are extremely faint Galactic globular clusters.

Numerous authors have shown that the predictions of $\Lambda \mathrm{CDM}$ simulations can be reconciled with the small number of observed MW dwarf galaxies if simple models for baryonic physical processes are taken into account when interpreting the results of numerical simulations (e.g., Bullock et al. 2001; Kravtsov et al. 2004; Simon \& Geha 2007). For example, Strigari et al. (2007) show that the central masses $\left(M_{0.6 \mathrm{kpc}}\right)$ of the MW dwarf galaxies are well constrained by the data and that their mass function closely matches the $M_{0.6 \mathrm{kpc}}$ mass function of both the earliest forming subhalos and the most massive accreted subhalos in the Via Lactea simulation.

A well-defined, deep survey of MW dwarf galaxies over a large fraction of the sky is critical to assess any of the above scenarios. The dwarf galaxies detected (or not) by such a survey will provide one of the best ways to rigorously test the $\Lambda \mathrm{CDM}$ paradigm by comparing a variety of metrics (distribution, mass, scale sizes, and number) of the MW dwarfs with the predictions of $\Lambda \mathrm{CDM}+$ galaxy formation models. Willman et al. (2002) and Koposov et al. (2008) have previously conducted automated searches for MW dwarfs in the SDSS and their corresponding detection limits. The original Willman et al. survey was only performed over a couple of thousand square degrees of sky. The Koposov et al. (2008) survey was performed with a more sensitive algorithm (critical, because they found many new satellites to be on the edge of detectability), but few galaxies were used to accurately quantify their detection limits.

In this paper, we present critical improvements to the present characterization of the detectability of MW dwarf galaxies over the $\sim 9500 \mathrm{deg}^{2}$ of the SDSS in Data Release 6 (DR6). We also present an improved detection algorithm over previous searches. We aim to construct the most complete, well-defined census of MW satellites by embarking on a MW all sky satellite hunt. This search will ultimately combine SDSS DR6 (Adelman-McCarthy et al. 2008), the Two-Micron All Sky Survey (2MASS; Skrutskie et al. 2006), and the upcoming Southern Sky Survey (Keller et al. 2007). The Southern Sky Survey will cover the entire $\sim 20,000$ $\operatorname{deg}^{2}$ below $\delta<0^{\circ}$ using the new Australian National University (ANU) SkyMapper telescope equipped with a $5.7 \mathrm{deg}^{2}$ widefield camera that is currently under construction, with survey operation expected to commence early 2009.

\section{SDSS DATA}

The SDSS (York et al. 2000) is an automated multicolor imaging and spectroscopic survey spanning $9500 \mathrm{deg}^{2}$ surrounding the North Galactic Pole. The $u, g, r, i$, and $z$ imaging data (Fukugita et al. 1996; Gunn et al. 1998) are photometrically and astrometrically reduced through an automatic pipeline (Hogg et al. 2001; Ivezić et al. 2004; Smith et al. 2002; Tucker et al. 2006; Pier et al. 2003). We subsequently correct for reddening with the Schlegel et al. (1998) extinction values given in the SDSS catalog. All following work is performed on point sources from DR6, using the photometry flags from the examples of database queries appropriate for point sources available on the
SDSS Skyserver Web site. ${ }^{4}$ To ameliorate effects of incompleteness in the point-source catalog and star/galaxy separation, we only consider sources brighter than $r=22.0$. The photometric data are provided by the SDSS DR6 (Adelman-McCarthy et al. 2008).

\section{SURVEY METHOD}

Low surface brightness MW satellites are detectable only by their resolved stars. With the least luminous known MW satellites, such as Boötes II, containing fewer than $\sim 20$ stars brighter than the main-sequence turnoff (MSTO; Walsh et al. 2007b), a deep, wide-field, uniform, multicolor photometric catalog is essential for searching for these objects. They will typically reveal their presence as statistically significant spatial overdensities relative to the Galactic foreground. Their signal can be enhanced by selecting stellar sources that are consistent in color-magnitude space with, for example, an old population of stars at a fixed distance. In this paper, we restrict ourselves to the old stellar populations characteristic of Local Group dSphs, but the population-specific elements of the algorithm can be easily modified for other systems. The strategy of our detection algorithm is built upon that of Willman et al. (2002) and Willman (2003) which utilized the photometric catalogs from SDSS and led to the discoveries of Ursa Major (Willman et al. 2005b) and Willman 1 (Willman et al. 2005a). It is also similar in spirit to Belokurov et al. (2007) and Koposov et al. (2008). Several systematic searches for MW dwarfs have also been done with non-SDSS data (Irwin 1994; Kleyna et al. 1997; Whiting et al. 2007).

In summary, our algorithm applies color and magnitude cuts to stars in the DR6 catalog, stores their distribution in a spatial array with $0.02 \times 0.02$ pixels, spatially smoothes the array with a Plummer surface density profile, and sets comprehensive thresholds for detection. Each of these steps is described in detail in the following sections.

\subsection{Data Management}

In order to efficiently manage thousands of square degrees of survey data in a catalog containing tens of millions of stars, we first divide the data set (in the case discussed in this paper, SDSS DR6) into stripes, each spanning $3^{\circ}$ in declination (to avoid projection effects) with $2^{\circ}$ of overlap in declination between adjacent stripes. This overlap creates a substantial redundancy to ensure that real objects are situated in the central $\sim 2^{\circ}$ of declination in at least one stripe, away from possible edgeeffects introduced at the stripe boundaries during the processing described in Section 3.3. We then take the longest continuous regions of the DR6 footprint in right ascension.

\subsection{Selection Criteria}

The mainly old, metal-poor stars of a nearby dwarf galaxy will occupy a well defined locus in the color-magnitude diagram (CMD), in contrast to MW stars which span a wide range in distance, age, and metallicity. Therefore selecting stars that are consistent in color-magnitude space with a population of old stars at a particular distance will significantly enhance the clustering contrast of a dwarf galaxy's stars over the foreground noise from MW stars.

We use theoretical isochrones in SDSS filters from Girardi et al. (2004) to define the regions of $(g-r, r)$ space likely to be

\footnotetext{
4 http://cas.sdss.org/dr6/
} 


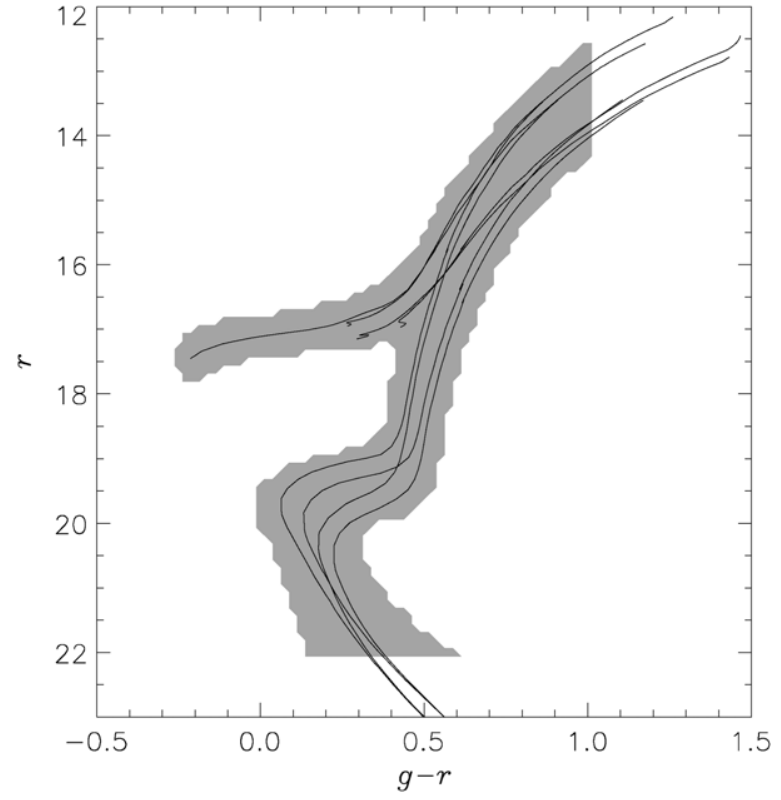

Figure 1. $(g-r, r) \mathrm{CMD}$ showing the two reddest and two bluest theoretical isochrones for old stellar populations $([\mathrm{Fe} / \mathrm{H}]=-2.27,-1.5$ and age $=8,14$ Gyr) at a distance modulus of $m-M=16.5(\sim 20 \mathrm{kpc})$, generated from Girardi et al. (2004). The shaded region shows pixels that pass the selection criteria.

populated by old, metal-poor stars. Simon \& Geha (2007) obtained spectra of stars in eight of the newly discovered dwarfsCVn, CVn II, Com, Her, Leo IV, Leo T, UMa, and UMa II-and found mean metallicities in the range $-2.29<[\mathrm{Fe} / \mathrm{H}]<-1.97$. Based on this result, we consider isochrones for populations with metallicities of $[\mathrm{Fe} / \mathrm{H}]=-1.5$ and -2.27 (the lower limit in Girardi et al. 2004) and with ages 8 and 14 Gyr. Four isochrones in these ranges can be used to bound the region of CMD space we are interested in, namely the four combinations of $[\mathrm{Fe} / \mathrm{H}]=-1.5$ and -2.27 and ages 8 and $14 \mathrm{Gyr}$. Figure 1 shows these four isochrones projected to a distance of $20 \mathrm{kpc}$.

We define the selection criteria by the CMD envelope inclusive of these isochrones $+/-$ the $1 \sigma(g-r)$ color measurement error as a function of $r$ magnitude. Shifting these isochrones over distances between $m-M=16.5$ and 24.0 in 0.5 mag steps defines 16 different selection criteria appropriate for old stellar populations between $d \sim 20 \mathrm{kpc}$ and $\gtrsim 630 \mathrm{kpc}$. We truncate our color-magnitude selection template at a faint magnitude limit of $r=22.0$, beyond which photometric uncertainties in the colors and star/galaxy separation limit the ability to detect these populations. We also truncate the selection template at $g-r=1.0$, as including redder objects adds more noise from MW dwarf stars than signal from more distant red giant branch (RGB) stars. Finally we do not include stars with $\delta g$ or $\delta r>$ $0.3 \mathrm{mag}$ in our analysis. To efficiently select stars within this CMD envelope, we treat the CMD as an image of $0.025 \times 0.125$ (color $\times$ mag) pixels and determine which stars fall into pixels classified as "good" according to the selection criteria. Figure 1 shows an example of the selection criteria, in this case for $m-M=16.5$ ( 20 kpc). The shaded region highlights pixels that would be classed as "good" for a system at $\sim 20 \mathrm{kpc}$.

\subsection{Spatial Smoothing}

After the photometric cuts are applied, we bin the spatial (R.A., decl.) positions of the selected stars into an array, $E$, with $0.02 \times 0.02$ pixel size. We use a locally defined coordinate
Table 1

Angular Sizes of the Satellites Detected in SDSS

\begin{tabular}{lc}
\hline \hline Object & $\begin{array}{c}r_{h} \\
\text { (arcmin) }\end{array}$ \\
\hline Boötes & 12.6 \\
Boötes II & 4.2 \\
Canes Venatici & 8.9 \\
Canes Venatici II & 1.6 \\
Coma Berenices & 6.0 \\
Hercules & 8.6 \\
Leo IV & 2.5 \\
Leo V & 0.8 \\
Leo T & 1.4 \\
Segue 1 & 4.4 \\
Ursa Major & 11.3 \\
Ursa Major II & 16.0 \\
Willman 1 & 2.3
\end{tabular}

system to avoid projection effects. We then convolve this twodimensional (2D) array with a spatial kernel corresponding to the expected surface density profile of a dSph. We refer to this smoothed spatial array as $A$. For our spatial kernel we use a Plummer profile with a 4.5 scale length. This value provides an effective compromise between the angular scale lengths of compact and/or distant objects with those of closer/more extended objects. For reference the angular sizes of the new satellites are listed in Table 1 . We use the $r_{h}$ values derived by Martin et al. (2008) except for Leo V (Belokurov et al. 2008).

The normalized signal in each pixel of $A$, denoted by $S$, gives the number of standard deviations above the local mean for each element:

$$
S=\frac{A-\bar{A}}{A_{\sigma}}
$$

The arrays of running means, $\bar{A}$, and running standard deviations, $A_{\sigma}$, are both calculated over a $0.9 \times 0.9$ window around each pixel of $A$. In particular, $A_{\sigma}$ is given by

$$
A_{\sigma}=\sqrt{\frac{n(A-\bar{A})^{2} * B-((A-\bar{A}) * B)^{2}}{n(n-1)}} .
$$

$B$ is a box filter with $n$ elements and is the same size as the running average window. The resulting array $A_{\sigma}$ gives the standard deviation value for each pixel of $A$ as measured over the $0.9 \times 0.9$ span of the filter. In the next section, we will define the detection threshold of this survey in terms of $S$, as well as in terms of the local stellar density $E$.

\subsection{Detection Threshold(s)}

In a large survey such as ours, it is critical to set detection thresholds strict enough to eliminate false detections but loose enough to retain known objects and promising candidates. To characterize the frequency and magnitude of purely random fluctuations in stellar density analyzed with our algorithm, we measure the maximum value of $S$ for $199,0005.5 \times 3^{\circ}$ simulated fields of randomly distributed stars that have been smoothed as described in the previous section. The only difference is that there is no gradient in stellar density across each field. In the interest of computational efficiency we do not use a running window for the mean and $\sigma$ of each simulated field. The field size is chosen such that 1000 fields roughly total an 


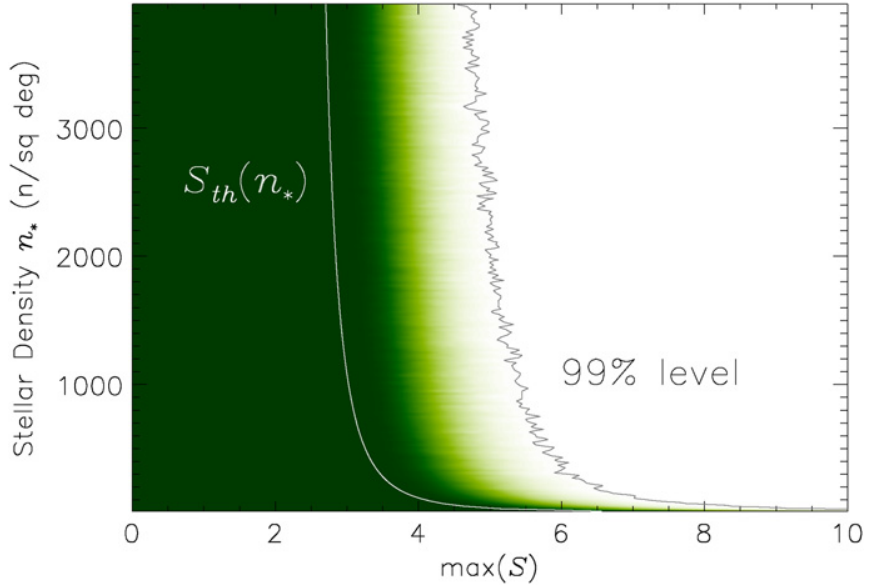

Figure 2. Two-dimensional cumulative histogram showing the distribution of $\max (S)$ values for smoothed fields for a range of stellar densities $n_{*}$. The gray line bounds $99 \%$ of the $\max (S)$ values and the white line shows our threshold density, $S_{\mathrm{th}}=f\left(n_{*}\right)$.

(A color version of this figure is available in the online journal.)

area equal to the DR6 footprint (neglecting regions lost during convolution). We select 199 stellar densities $n_{*}$ to simulate linearly spaced between 10 and 4000 stars per $\mathrm{deg}^{2}$. This range of stellar densities is to model the density range we find after applying the color-magnitude selection criteria described in Section 3.2 across the SDSS. In Section 5.1, we study the variation of detection limits with Galactic latitude (foreground stellar density); the typical number densities we will consider there are higher than 10-4000 stars per $\mathrm{deg}^{2}$, because we wish to parameterize the detection limits in terms of the density of all stars bluer than $g-r=1.0$ and brighter than $r=22$.

Figure 2 shows a two-dimensional cumulative histogram of the 199,000 $\max (S)$ values over a range stellar densities. In low density fields, the distribution of pixel values becomes nonGaussian so a simple, global threshold value is insufficient. The solid gray line shows the contour containing $99 \%$ of the 199,000 $\max (S)$ values at each density. If we simply used a value like this as our threshold, we would be biasing ourselves against detecting extended objects; large angular scale length systems may not have a peak pixel value above this value, for example because stars in the object itself increases the local running mean and sigma. However, such an overdensity may have some characteristic area larger than any random fluctuation.

We thus define a detection threshold based on both the peak density and a characteristic area of an overdensity. To define such an area we scale down the 99\% contour from Figure 2 and define a threshold density $S_{\text {th }}\left(n_{*}\right)$ as a function of stellar number density (white line in Figure 2). Then, using the 199,000 random fields we examine the relationship between the peak density $\max (S)$ divided by $S_{\mathrm{th}}\left(n_{*}\right)$ and "detection" area, i.e., the area of contiguous pixels of $S$ that have values above $S_{\text {th }}\left(n_{*}\right)$ (the white line). Figure 3 shows this area versus $\max (S) / S_{\mathrm{th}}\left(n_{*}\right)$ for the random fields. If we assume a purely random foreground, we would expect one false detection in the DR6 footprint above an area of $\sim 55 \operatorname{arcmin}^{2}$ or above a peak density of $\sim 1.6 \times S_{\text {th }}\left(n_{*}\right)$. These numbers are set by the factor by which we scale down the threshold function and are themselves arbitrary.

Based on the results of these simulations we set the area threshold to a more conservative $60.0 \mathrm{arcmin}^{2}$ and the density threshold to a more conservative $1.75 \times S_{\mathrm{th}}\left(n_{*}\right)$ to eliminate false positive fluctuations while preserving all of the known objects

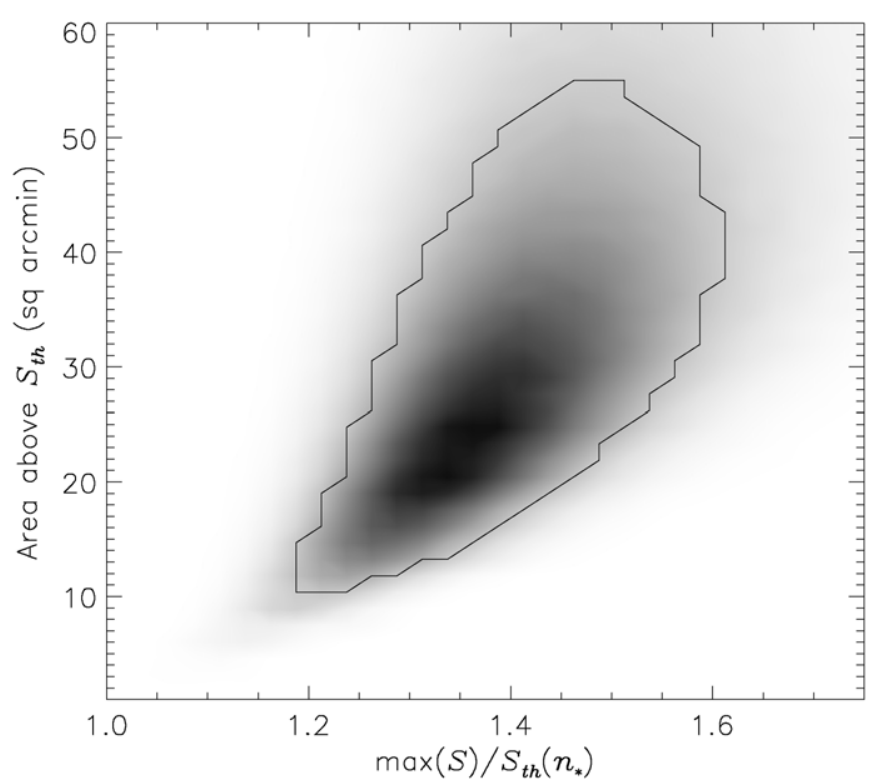

Figure 3. "Detection" area vs. $\max (S) / S_{\mathrm{th}}\left(n_{*}\right)$ for the 199,000 random fields. The black contour shows the level at which purely random clustering would produce one false detection over the approximate area of DR6.

within DR6, including Boötes II (Walsh et al. 2007a) and the Koposov 1 and 2 globular clusters (Koposov et al. 2007). Thus, a detection is defined as a region where

1. the area of a group of contiguous pixels of $S$ above $S_{\mathrm{th}}\left(n_{*}\right)$ (white line, Figure 2) is greater than $60.0 \operatorname{arcmin}^{2}$ or

2. any single pixel of $S$ is greater than $1.75 \times S_{\mathrm{th}}\left(n_{*}\right)$.

We implement these adaptive density thresholds as a function of local stellar density $n_{*}$, so that the algorithm may be run over large fields with varying density and allow direct comparison between fields of greatly different densities. The stellar density $n_{*}$ is calculated for each pixel of the smoothed, normalized, spatial array $S$, as the $0.9 \times 0.9$ running average of the original spatial density array $E$.

To summarize our algorithm:

1. Apply CMD cuts, bin spatial positions of remaining stars into $E$.

2. Smooth $E$ with Plummer profile to get $A$.

3. Calculate the $0.9 \times 0.9$ running mean $\bar{A}$ and running standard deviation $A_{\sigma}$.

4. Define $S$ as $S=(A-\bar{A}) / A_{\sigma}$.

5. Calculate array of threshold values $S_{\text {th }}$ as a function of stellar density $n_{*}$ (from $0.9 \times 0.9$ running mean of $E$ ).

6. Detections are where contiguous regions of pixels with $S>S_{\mathrm{th}}\left(n_{*}\right)$ are greater than $60.0 \mathrm{arcmin}^{2}$ or any single pixel is greater than $1.75 \times S_{\mathrm{th}}\left(n_{*}\right)$.

\subsection{Identifying and Evaluating Detections}

For each of our DR6 data strips defined in Section 3.1, the steps outlined in the previous sections are repeated in $0.5 \mathrm{mag}$ distance modulus intervals, and these 16 frames are layered to form a three-dimensional (3D) array. This 3D approach eliminates complications with multiple detections of a single object using selection criteria for different distance moduli, and selects out the strongest detection. The coordinates of stars within each detection and the CMD within the detection's area are plotted for later visual inspection. Galaxy clusters and point sources around partially resolved background galaxies 


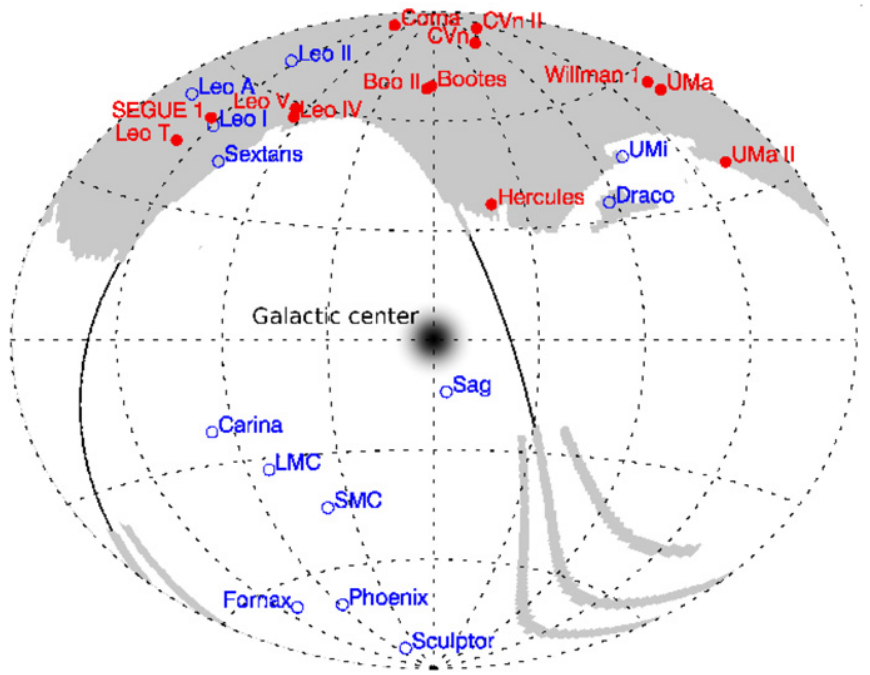

Figure 4. Aitoff projection of the DR6 footprint in Galactic coordinates, centered on the Galactic center. Previously known dwarfs are marked with open blue circles, satellites discovered in SDSS are marked with filled red circles.

(A color version of this figure is available in the online journal.)

(such as their associated globular clusters) will contaminate the detections, but these can be identifiable based on their CMDs (see Section 4), leaving a list of potential new MW satellite galaxies and globular clusters. At this point, follow-up observations are typically necessary to confirm the existence and nature of these candidates.

\section{APPLICATION TO SDSS DATA RELEASE 6}

We apply our search algorithm (as described in Section 3) to $21,439,777$ sources with $r<22.0$ and $g-r<1.0$ in the $9500 \mathrm{deg}^{2}$ of imaging data in DR6 of the SDSS. The DR6 footprint is shown in Figure 4, along with previously known dSphs (open blue circles) and satellites discovered in SDSS (closed red circles).

The significance of our detections of known objects in terms of their peak density and area is shown in Figure 5. In the total area of DR6 analyzed, we find 100 unique detections above the thresholds, defined by the dotted lines of Figure 5 . The positions of each of these detections are cross-referenced against the SIMBAD database ${ }^{5}$ as well as visually inspected via the SDSS Finding Chart Tool. ${ }^{6}$ Of our 100 detections, 19 are MW/Local Group dwarfs (counting Boötes II, Willman 1 and Segue 1), 17 are Galactic globular clusters (including Koposov 1 and 2), two are known open clusters, 28 are clusterings of point sources associated with background galaxies such as unresolved distant globular clusters, and four are Abell galaxy clusters. The remaining 30 do not correspond to any cataloged objects, but CMDs of only a handful of these are consistent enough with a faint MW satellite to warrant follow-up. The remainder may be galaxy clusters whose detected center differs from its cataloged center by more than $\sim 0.25$, or perhaps tidal debris. If the MW stellar halo is the result of accretion of dSph then evidence of this accretion is expected. It should be noted that objects with relatively large angular size, such as Draco and Sextans, substantially increase the average stellar density of the area they

\footnotetext{
5 http://simbad.u-strasbg.fr/simbad/

6 http://cas.sdss.org/astrodr6/en/tools/chart/chart.asp
}

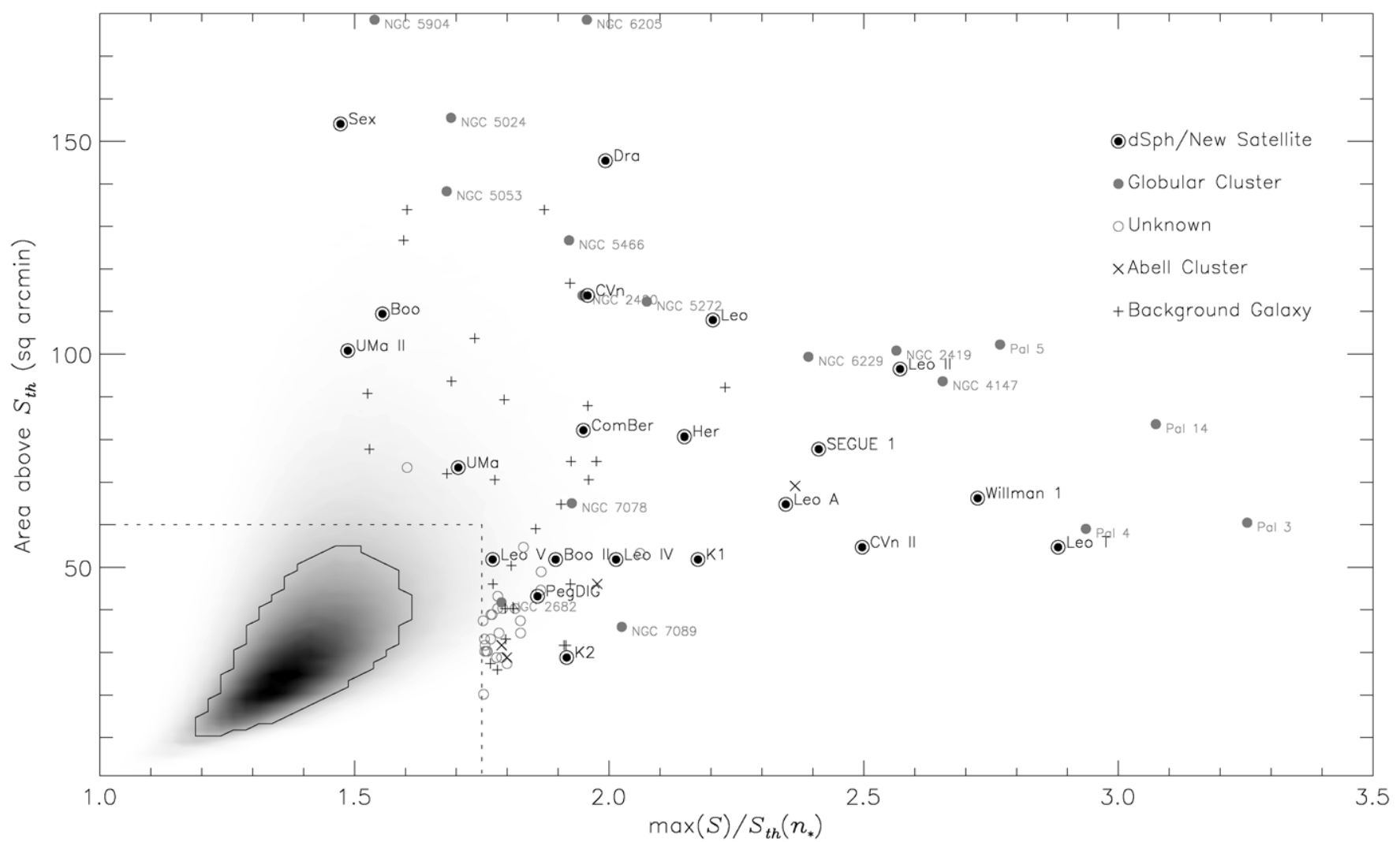

Figure 5. Same as Figure 3 but showing all detections in DR6. Dotted black lines show the adopted thresholds. Galactic/Local Group dSphs and Koposov 1 and 2 are shown as black filled circles. The brightest objects such as Draco and Sextans increase the average stellar density of the area they occupy, which increases $S_{\text {th }}\left(n_{*}\right)$ in their vicinity. This means that $\max (S) / S_{\mathrm{th}}\left(n_{*}\right)$ is not as high as one might expect. 


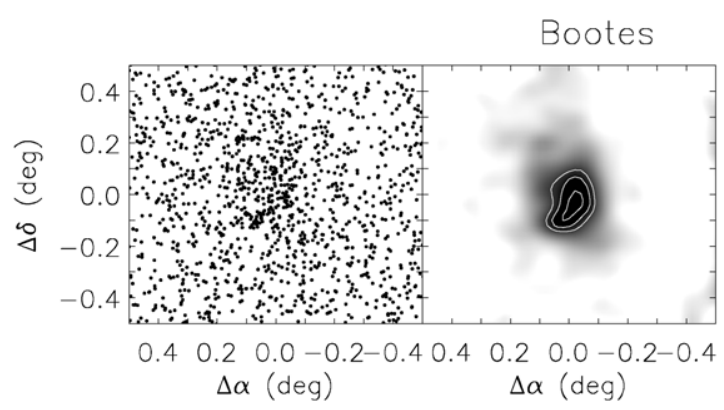

$M_{v}=-6.3, d=66 \mathrm{kpc}$
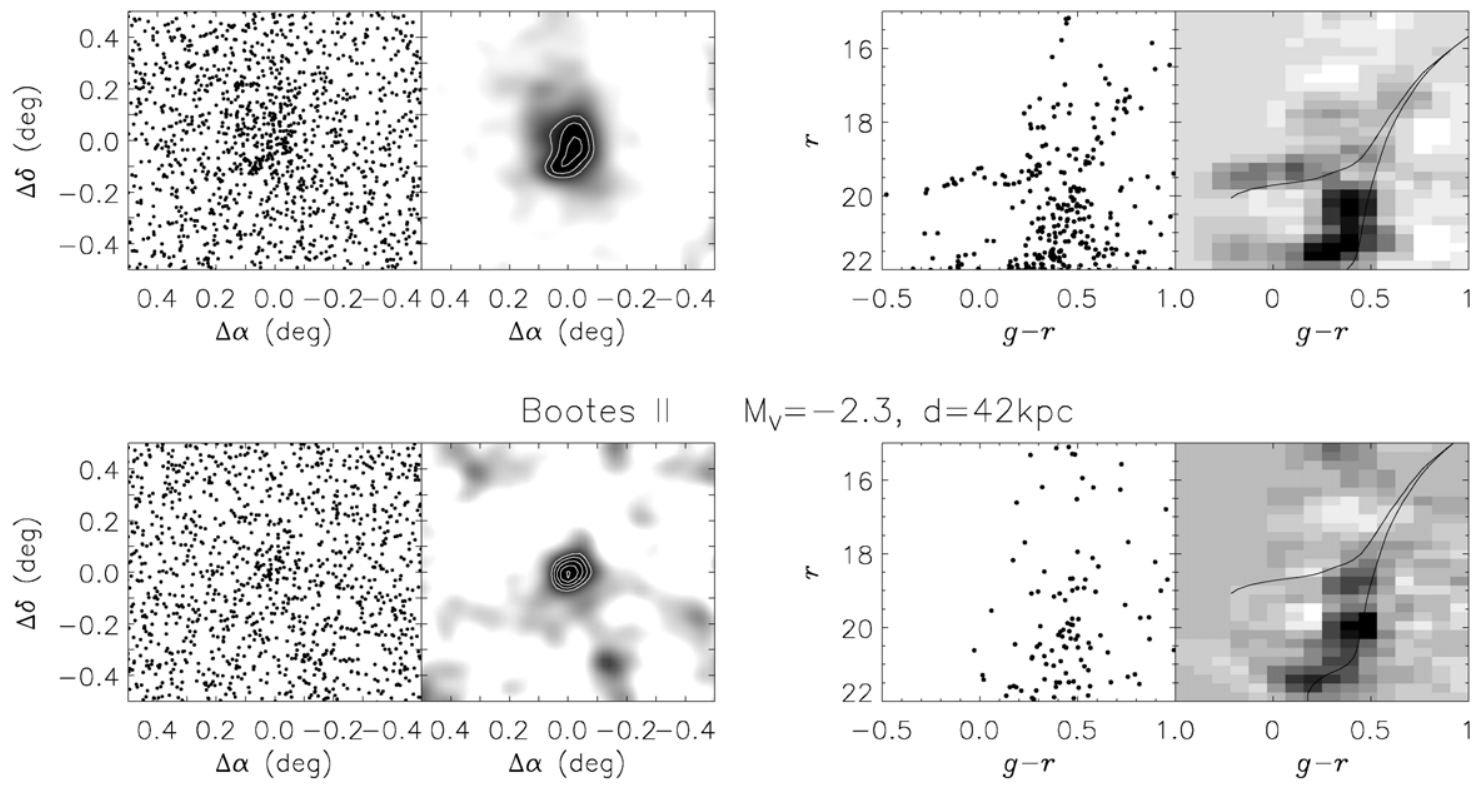

$M_{\mathrm{v}}=-2.3, \mathrm{~d}=42 \mathrm{kpc}$

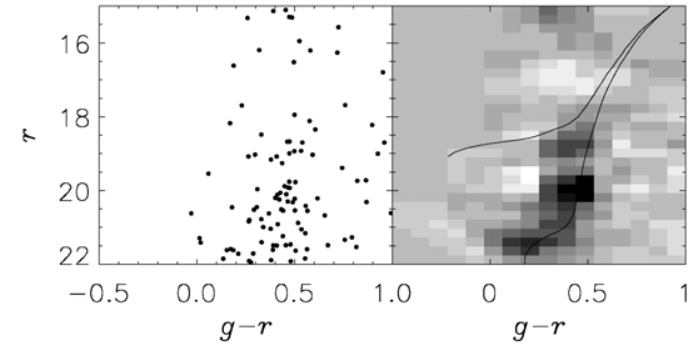

Canes Venatici
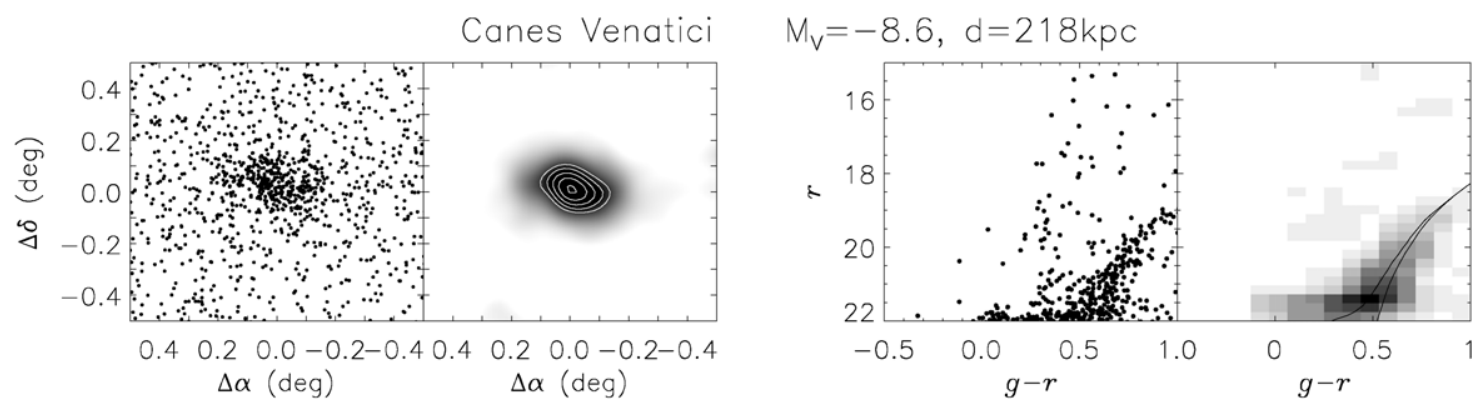

Canes Venatici II

$M_{v}=-4.9, d=160 \mathrm{kpc}$
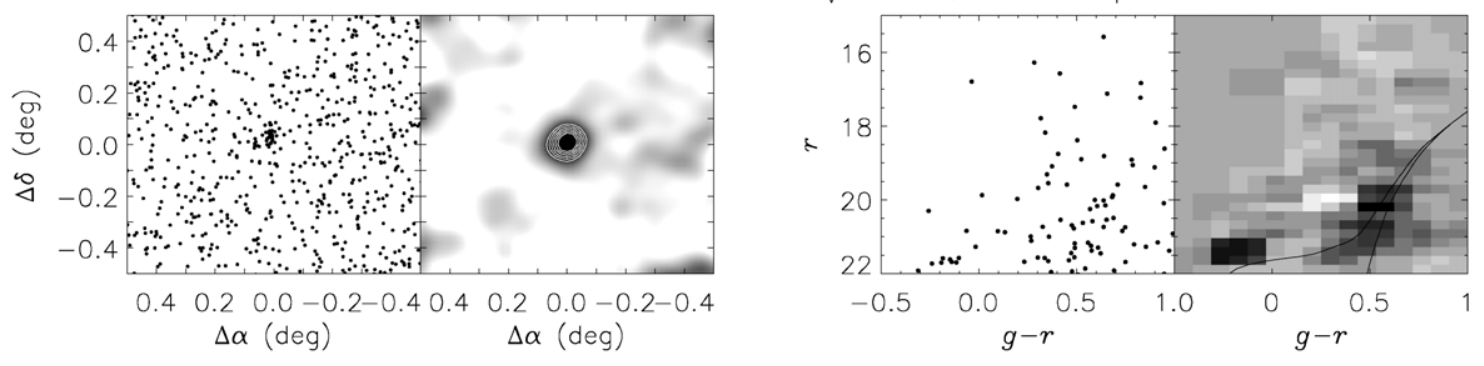

Coma Berenices

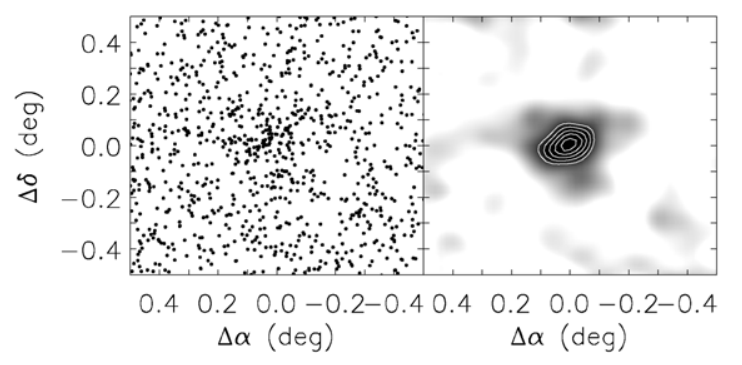

$M_{\mathrm{V}}=-4.1, \mathrm{~d}=44 \mathrm{kpc}$

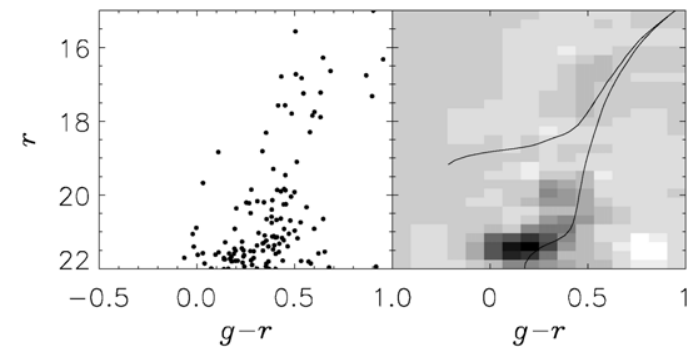

Figure 6. Our detections of recently discovered MW satellites. Left: spatial plot of sources passing selection cut. Middle left: contour of smoothed spatial plot. Contours show 1.0, 1.2, 1.4, 1.6, 1.8, and 2.0 times the density threshold. Middle right: CMD of region enclosed by contours. Right: Hess diagram of the same region, with $13 \mathrm{Gyr},[\mathrm{Fe} / \mathrm{H}]=-2.3$, Girardi et al. (2004) isochrone at the object's distance overplotted.

occupy which increases the threshold density, meaning they are not as high above the density threshold as one might expect. Due to the area threshold however, they are still very prominent detections.
We recover all of the newly discovered objects that are within DR6 and the "classically" known Draco, Leo, Leo II, Leo A, Sextans, and Pegasus DIG dwarfs. Our detections of the new dwarfs are presented in Figures 6-8. These figures 


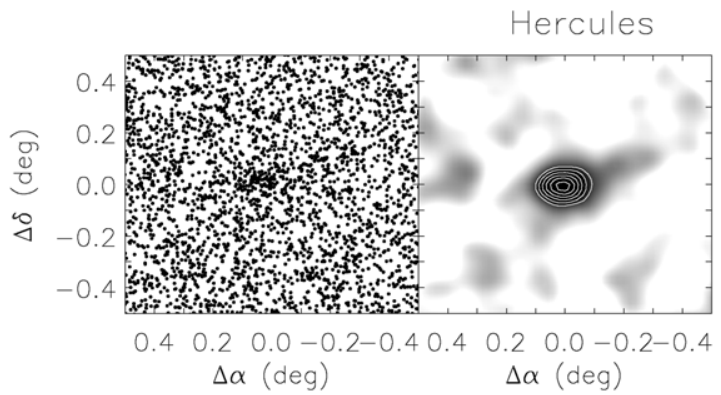

$M_{\mathrm{V}}=-6.6, \quad d=132 \mathrm{kpc}$
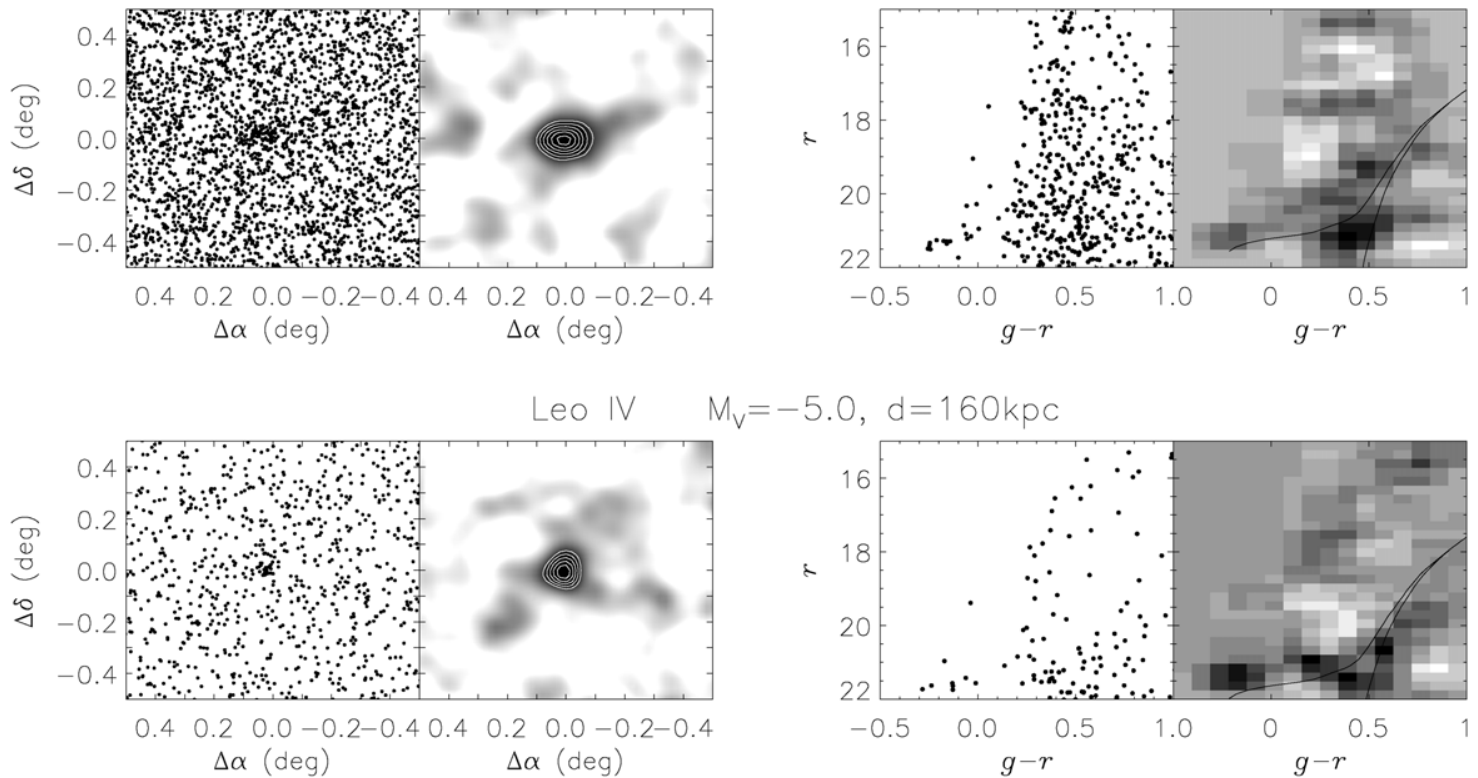

$M_{v}=-5.0, d=160 \mathrm{kpc}$
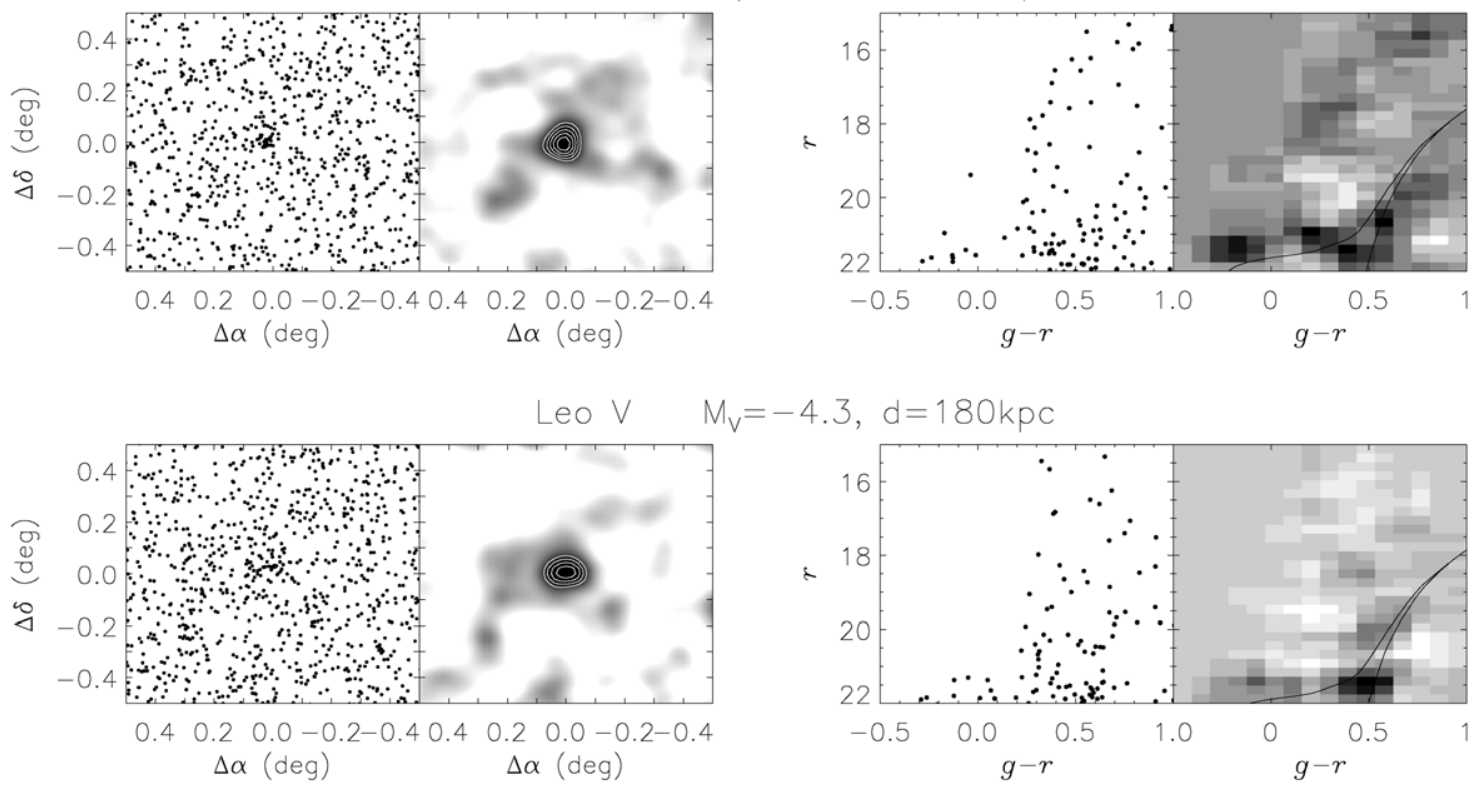

$M_{v}=-4.3, d=180 \mathrm{kpc}$
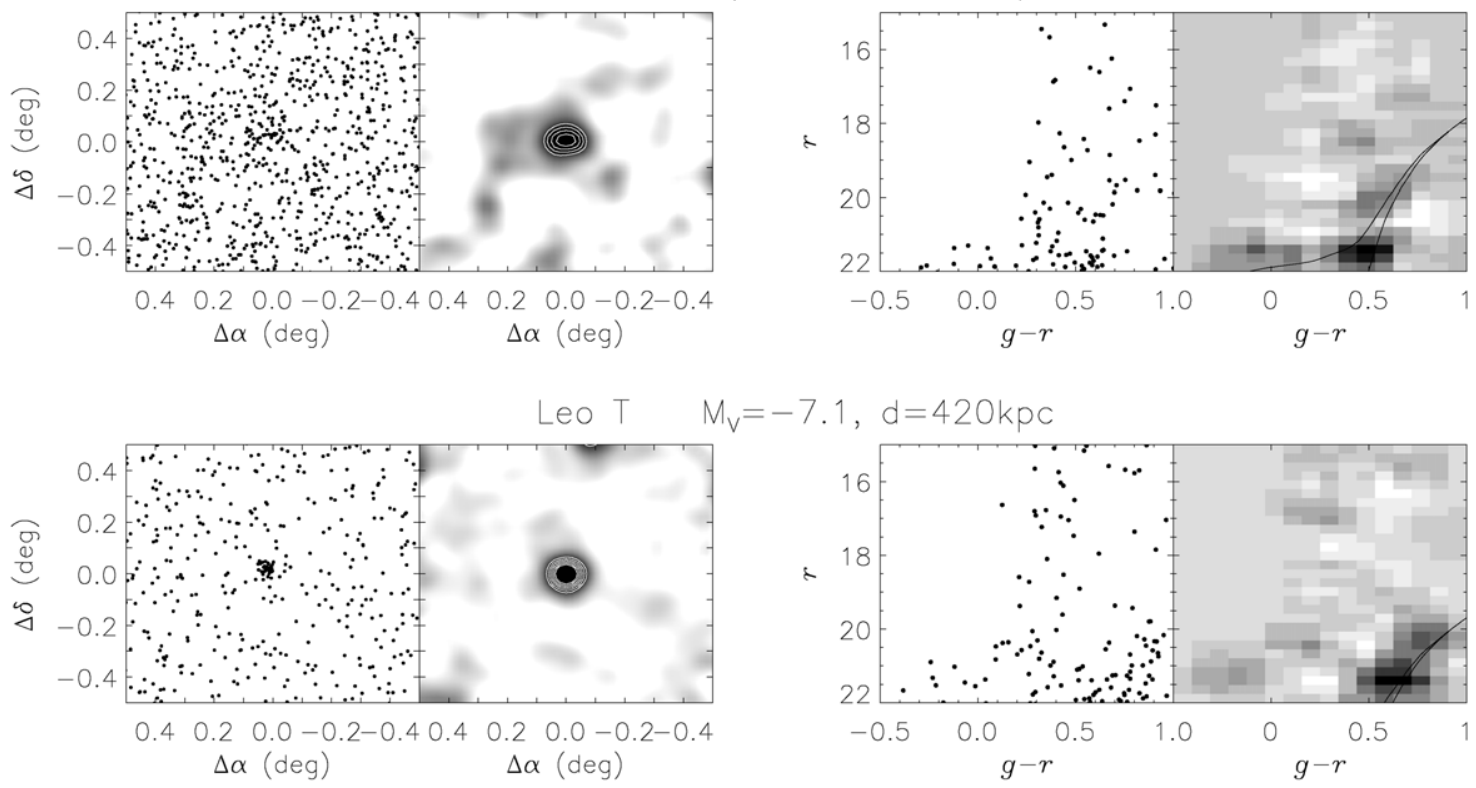

$M_{V}=-7.1, \quad d=420 \mathrm{kpc}$
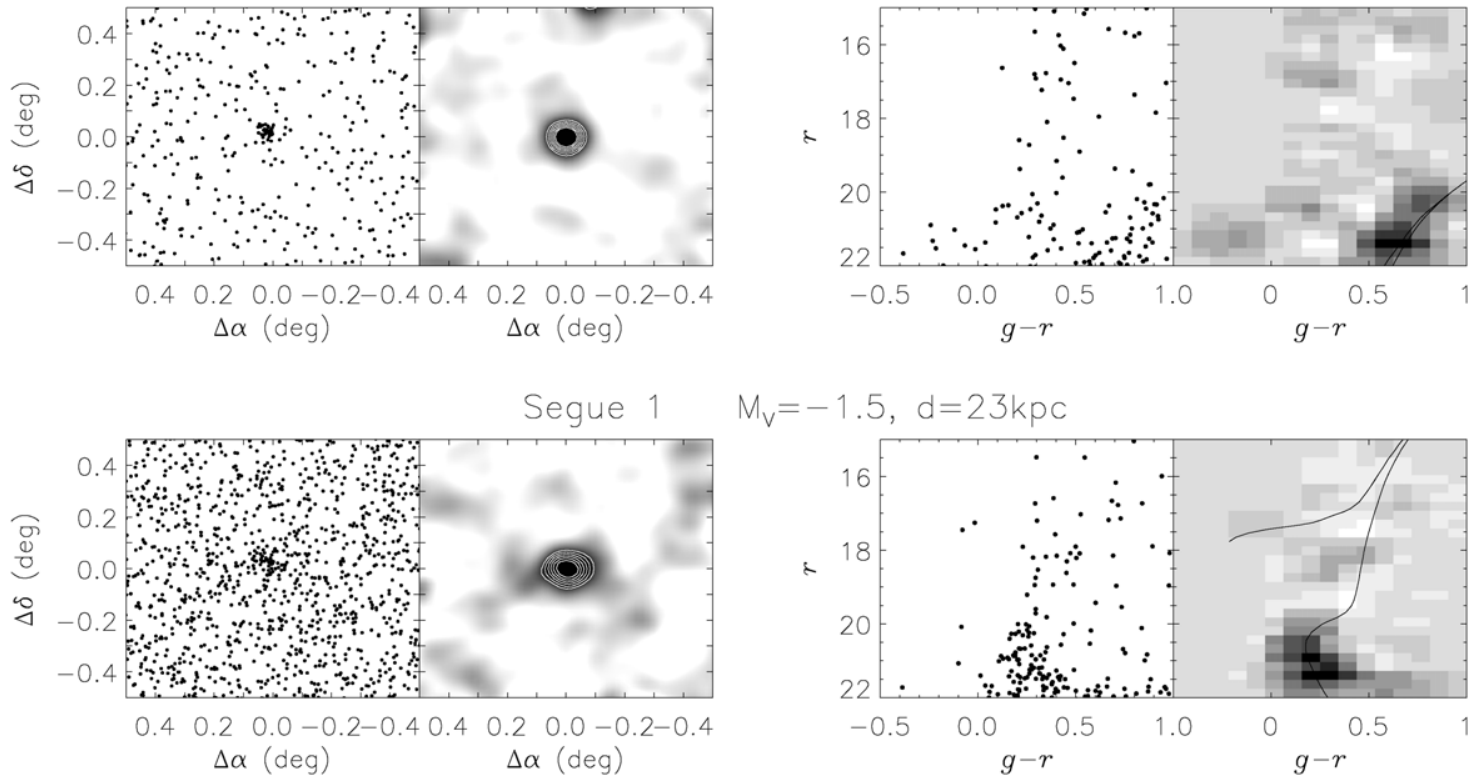

$M_{v}=-1.5, d=23 k p c$

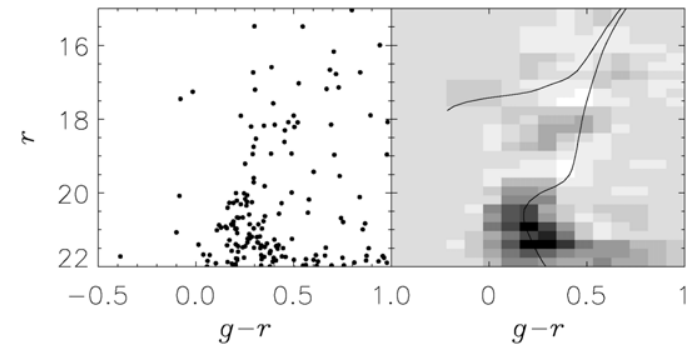

Figure 7. Detections of recently discovered MW satellites cont.

are identical to those output by the automated algorithm for each detection, aside from the addition of figure titles $\left(M_{V}\right.$ and distances from Martin et al. 2008 and references therein). The left panel shows the spatial positions of stars passing the photometric selection criteria at the distance modulus at which the object was most strongly detected. The middle-left panel shows the contour plot corresponding to $S$, where the contour levels are $S / S_{\mathrm{th}}\left(n_{*}\right)=1.0,1.2,1.4,1.6,1.8$, and 2.0. The middleright panel is the CMD of the detection area and the right panel is the field-subtracted Hess diagram. The isochrone is that of 

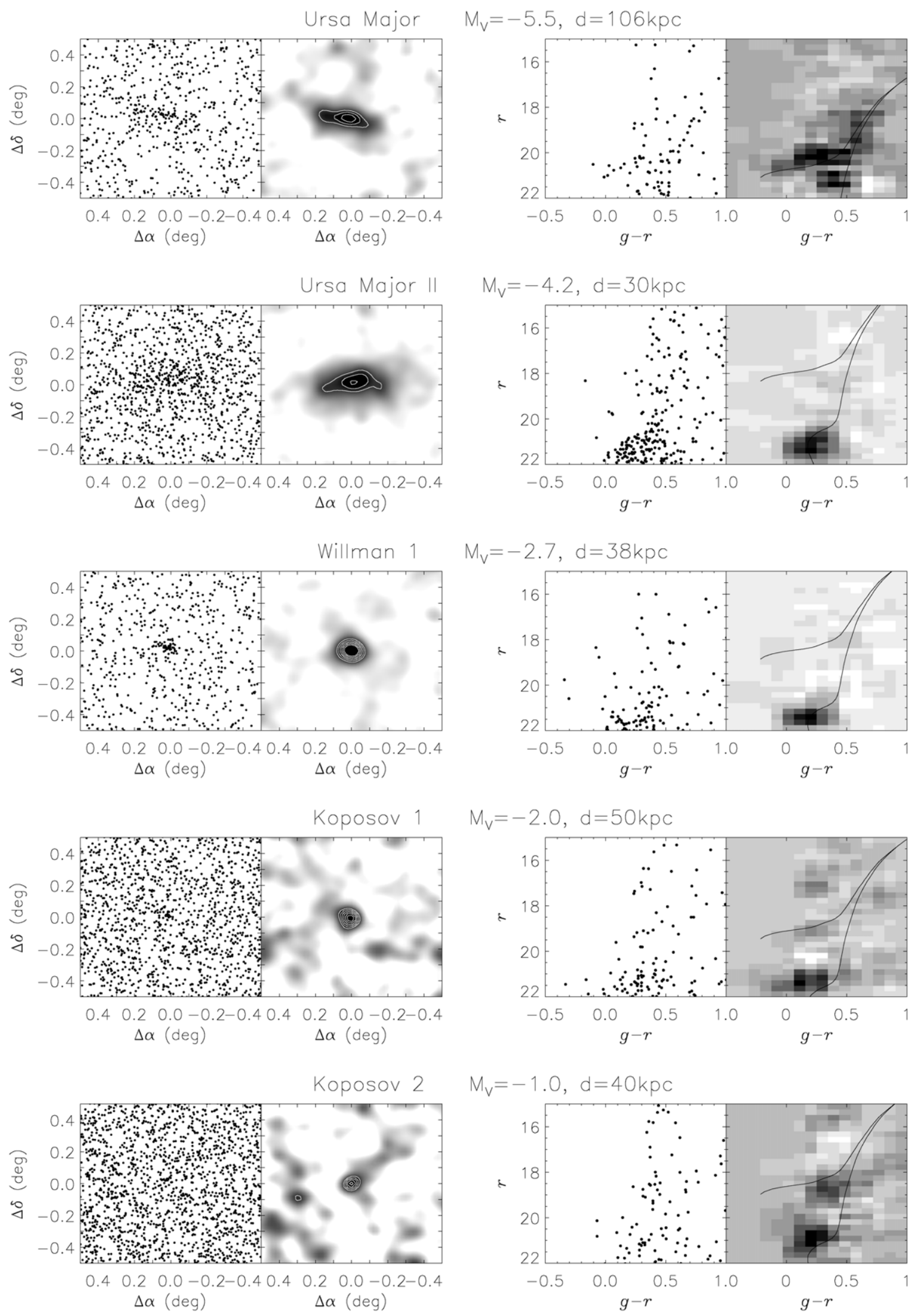

Figure 8. Detections of recently discovered MW satellites cont.

a $13 \mathrm{Gyr},[\mathrm{Fe} / \mathrm{H}]=-2.3$ from Girardi et al. (2004) at the distance specified. Besides demonstrating the effectiveness of our algorithm, these detections provide a benchmark with which to compare candidates and to determine which are consistent with being a new dwarf satellite.
To further illustrate the product of our algorithm we also show examples of undesired detections in Figure 9: the galaxy cluster Abell 1413 (top) and Virgo cluster galaxy NGC 4486 (bottom). These represent typical detections of background galaxies and galaxy clusters. 

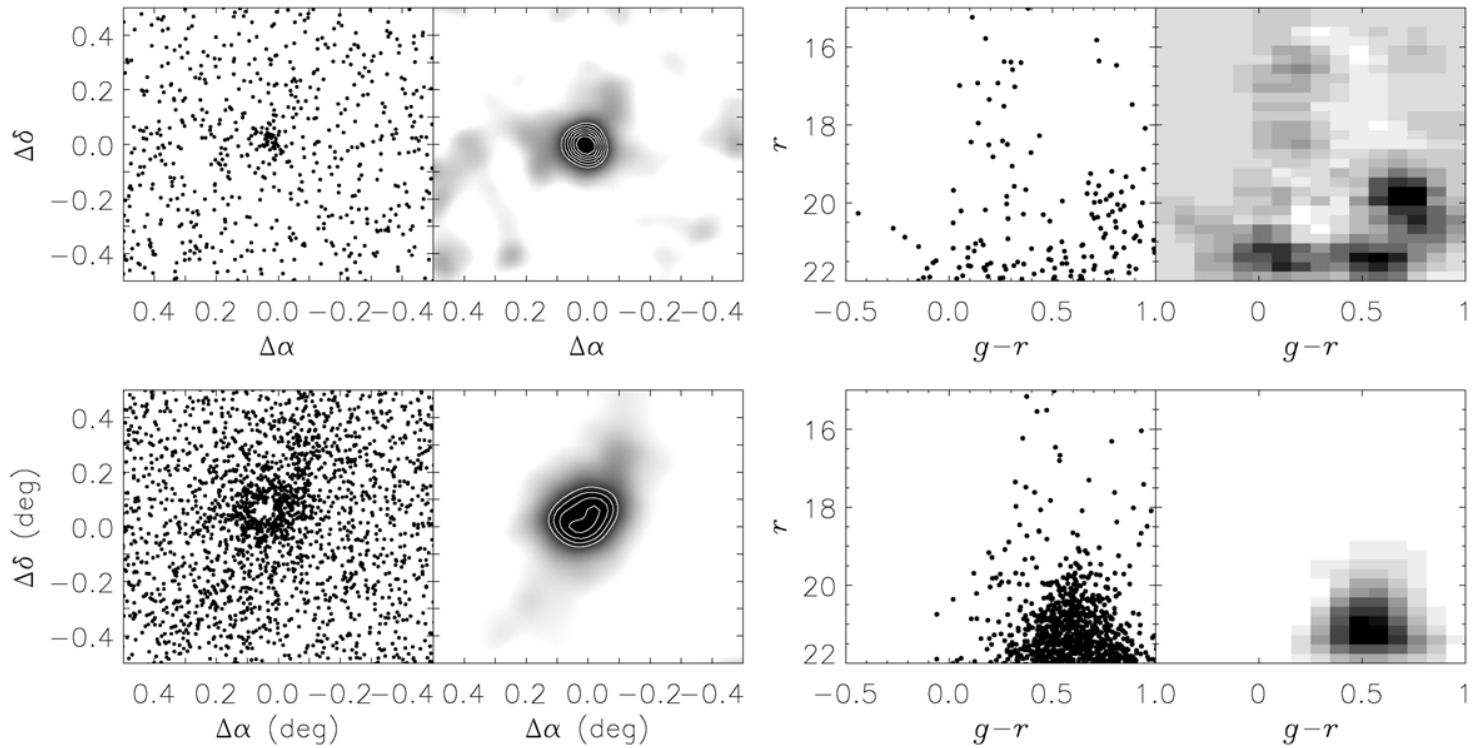

Figure 9. Detections of Abell 1413 (top) and NGC 4486 (bottom) as examples of galaxy cluster and background galaxy detections.

Table 2

Positions of Strongest MW Satellite Candidates

\begin{tabular}{lcccc}
\hline \hline Designation & $\alpha$ & $\delta$ & $(\alpha, \delta)$ & $D(\mathrm{kpc})$ \\
\hline CVn W & $13: 16: 04.8$ & $+33: 15: 00$ & $(199.02,33.25)$ & $\sim 160$ \\
Her X & $16: 27: 45.6$ & $+29: 27: 00$ & $(246.94,29.45)$ & $\sim 100$ \\
UMa Y & $12: 11: 57.6$ & $+53: 35: 24$ & $(182.99,53.59)$ & $\sim 100$ \\
Vir Z & $12: 20: 19.2$ & $-1: 21: 00$ & $(185.08,-1.35)$ & $\sim 40$ \\
\hline
\end{tabular}

\subsection{Candidate Milky Way Satellites}

Figure 10 shows four unidentified overdensities that have CMDs qualitatively similar to that of a dSph. All show statistically significant spatial clustering and do not coincide with a visible overdensity of background galaxies. While several unknown detections have CMDs broadly consistent with old stellar populations, we present here four detections that are as strong as or stronger than the detections of UMa and PegDIG. We present their positions and distances estimated from the CMD in Table 2.

\section{EXPLORING DETECTION EFFICIENCY WITH SYNTHETIC SATELLITES}

The most advantageous aspect of a large, uniform search for MW dwarfs is the ability to rigorously calculate its detection limits in order to compare observations with predictions. To calculate the detection completeness of our search, artificially generated galaxies are embedded in simulated stellar foreground fields and put through the detection algorithm to investigate the sensitivity as a function of galaxy distance, luminosity, scale length, and Galactic latitude. In this section, we describe in detail the method used to synthesize artificial SDSS fields and dSph satellites.

\subsection{Sowing the Simulated Fields}

When simulating fields in which to embed artificial galaxies, our goal is to create a large number of fields with the same pointsource color, magnitude, and density distributions as observed in the SDSS DR6 footprint. The detectability of a dSph may change depending on its position in the sky. For example, those at low Galactic latitudes will be harder to detect than those at high latitudes, owing to the greater number of foreground stars. The relative proportions of the thin disk, thick disk, and stellar halo will also vary with latitude and longitude, changing what fraction of foreground stars will be included in the colormagnitude selection described in Section 3.

To conduct a controlled experiment to see how Galactic foreground affects detection efficiency over the DR6 footprint, we first select three fiducial latitudes to simulate: the median latitude of the DR6 footprint, and the latitudes above and below which $10 \%$ of the survey lies. Figure 11 shows the fraction of sky observed by SDSS DR6 as a function of latitude (dashed line). Weighting this fraction by $\cos b$ gives the relative area on the celestial sphere that each observed latitude occupies (solid gray line), showing that the majority of the DR6 footprint by area is located between $b \approx 45^{\circ}$ and $b \approx 65^{\circ}$. The cumulative total (solid black line) allows us to choose the latitudes corresponding to $10 \%, 50 \%$, and $90 \%$ levels of DR6, namely $31^{\circ}, 53^{\circ}$, and $73^{\circ}$. These are the three values of latitude that we implement in our simulations.

Now that we have chosen what latitudes to simulate, we need to relate these to the stellar foreground density. Figure 12 presents a 2D histogram of latitude and foreground density, considering only stars brighter than $r=22.0$ and bluer than $g-r=1.0$. This figure shows a span in foreground levels at each latitude. The solid black line traces the median and our chosen latitudes are marked along the $x$-axis. For each of our latitudes, we take a slice through the $2 \mathrm{D}$ histogram and use this distribution of densities to randomly assign a density for each of our simulated fields. Each artificial star in our simulated fields is assigned photometric parameters from a star in DR6, chosen at random from all stars within \pm 0.5 of the latitude in question. These stars are then randomly distributed in a $3^{\circ} \times 3^{\circ}$ field. Although the true distribution of point sources in the SDSS is certainly not random, this approach allows us to perform a well controlled experiment to derive the detectability of dwarfs in the limit of a purely random point-source distribution, using many realizations of the data and of simulated galaxies. Willman (2003) showed that using real SDSS data rather than randomized realizations of SDSS data to derive the detectability of dwarfs results in detection limits that are more conservative 

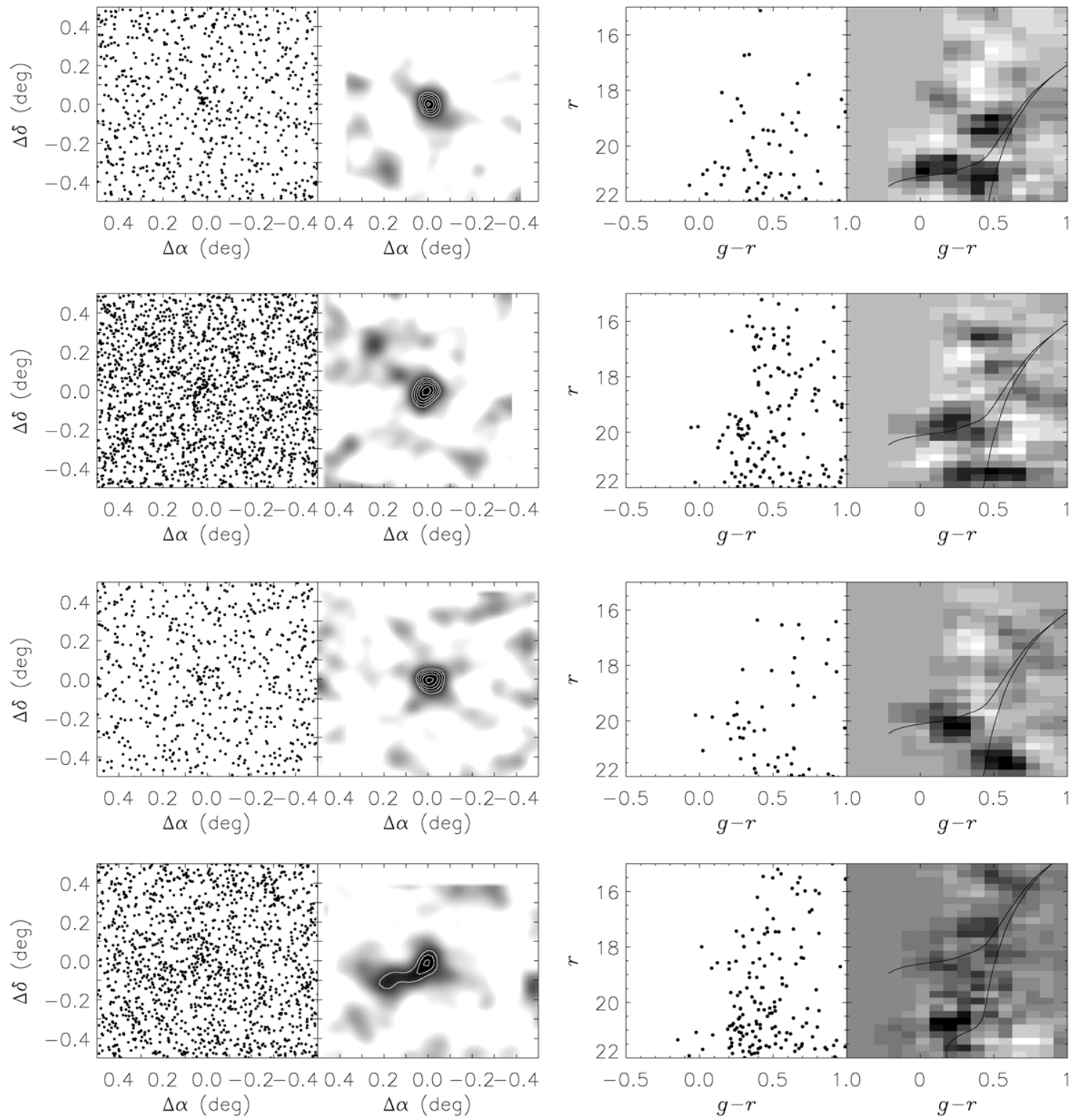

Figure 10. Four unknown detections with detection strength equal to or greater than known satellites (except Leo V). From top to bottom: Canes Venatici W, Hercules $\mathrm{X}$, Ursa Major Y, and Virgo Z. Isochrones show the distance interval at which these overdensities produced the strongest detections.

by $0.0-0.3$ mag than those derived from randomized fields, for the 4.5 smoothing filter used in this paper. We may thus overestimate the detectability of dwarfs by a couple tenths of a dex in magnitude.

\subsection{Forging Virtual Dwarfs}

To simulate a dSph galaxy CMD, we enlist Hubble Space Telescope (HST) observations of three MW satellites ${ }^{7}$ : Carina, Draco, and Ursa Minor (Holtzman et al. 2006). Figure 13 shows the combined CMD of these objects. We take this $M_{V}$ and $V-I \mathrm{CMD}$ and translate it in color and magnitude loosely to match the Girardi et al. (2004) isochrones in $g$ and $r$. The reasonable agreement between the $H S T$ data after approximate transformation and Girardi et al. (2004) isochrones in $g$ and $r$ is sufficient to allow the use of the HST data for our simulated objects.

\footnotetext{
7 http://astronomy.nmsu.edu/holtz/archival/html/lg.htm
}

We use these data to create a composite old stellar population catalog of stars brighter than $M_{r}=6$ by combining sources from the three HST dwarfs. Carina, Draco, and Ursa Minor each contribute 5,548, 4,487, and 3,296 stars, respectively. Each time we simulate a dwarf galaxy of $x$ stars, we select those $x$ stars at random from this composite catalog. The luminosity is calculated from the integrated flux from all stars, and a correction added to account for stars below an absolute magnitude of $M_{r}=6$. The cumulative luminosity functions in the right panel of Figure 13 show that typically $\sim 10 \%$ of the total flux originates from stars below this cutoff. We then adjust the photometry of the stars to the correct distance modulus and add photometric scatter to reflect increasing measurement uncertainty with fainter magnitudes. We do this by finding the best fit for the $1 \sigma$ magnitude uncertainty as a function of $g$ and $r$ magnitude in the SDSS data, and adding a normally distributed random realization $\delta$ of this value $\sigma(m)$ for the adjusted magnitude, $m_{\text {star }}=m+\delta \sigma(m)$. We then assign random 


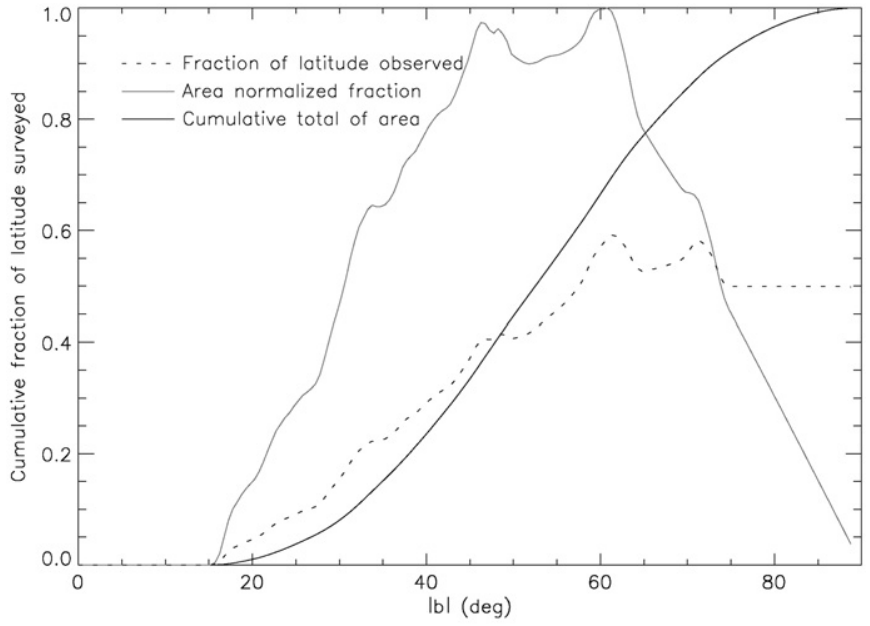

Figure 11. Fraction of sky as observed by SDSS DR6 at each latitude. The dotted line shows what fraction of the small circle on the celestial sphere traced by each latitude has been surveyed, and the gray line is this fraction weighted by the cosine of latitude, to give a relative sky area observed at each latitude. The largest area of DR6 observations occur at $b \simeq 60^{\circ}$. The solid black line is the cumulative total of the gray line.

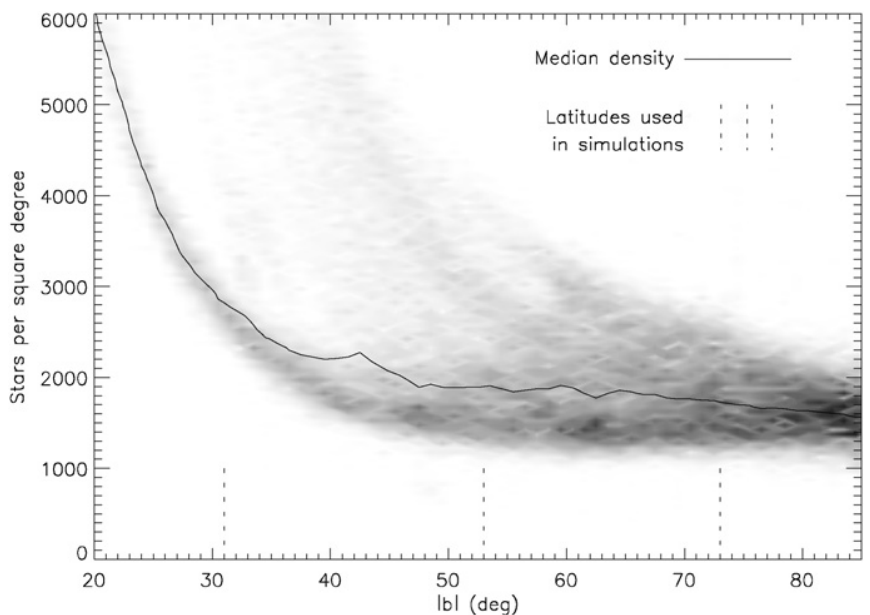

Figure 12. Gray-scale plot of the number of sources per deg ${ }^{2}$ satisfying $r<22.0$ and $g-r<1.0$ vs. absolute Galactic latitude. The black line traces the median density at each latitude. The three latitudes we choose to simulate are marked along the $x$-axis.

positions based on a Plummer surface brightness profile with a specified physical scale length at a given heliocentric distance.

Figure 14 shows examples of three simulated dSphs. The middle panel shows a system not unlike Boötes II, highlighting the paucity of stars in the objects we are searching for. It is important to note that at these low luminosities, the total luminosities of galaxies with the same number of stars can vary dramatically, with this variation increasing for galaxies with fewer stars. A single RGB star can have a magnitude of $M_{r}=-3$ which is well in the regime of the total magnitude of recently discovered satellites. Each generated galaxy is embedded in a simulated field, and then processed as described in Sections 3.2-3.4.

\subsection{Charting Detection Efficiency}

To test the efficiency of our search algorithm as a function of galaxy luminosity, scale length, distance, and Galactic latitude, we generate a total of 3,825,000 galaxies spanning ranges in Galactic latitude, luminosity, physical size, and distance. We

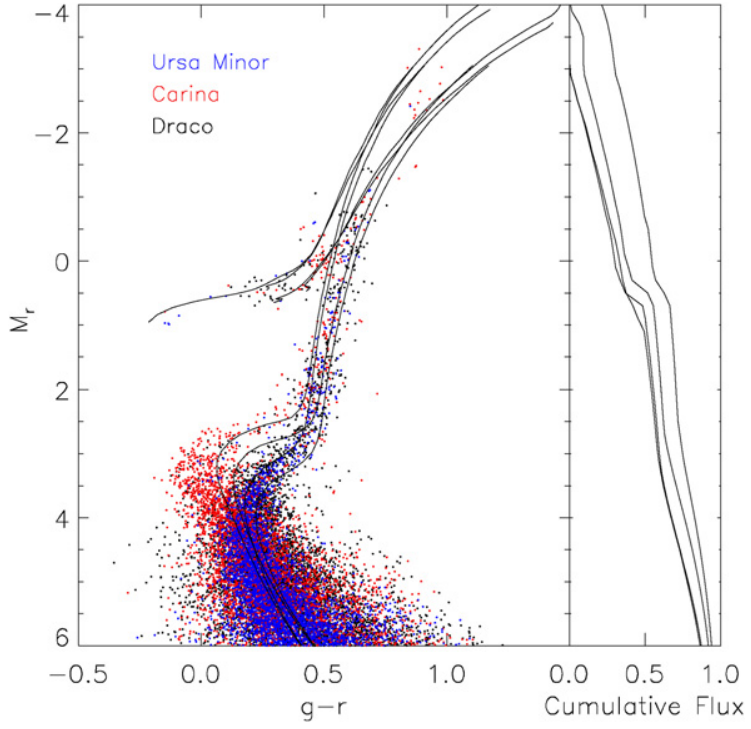

Figure 13. HST data of three MW satellite dSphs (Carina: red, Draco: black, and Ursa Minor: blue; Holtzman et al. 2006) with SDSS isochrones (Girardi et al. 2004) overlaid. The right panel shows the cumulative luminosity functions for the corresponding isochrones, using the four combinations of $[\mathrm{Fe} / \mathrm{H}]=$ $-2.27,-1.5$ and age $=8,14$ Gyr. Data are corrected for distance and presented in absolute magnitude.

(A color version of this figure is available in the online journal.)
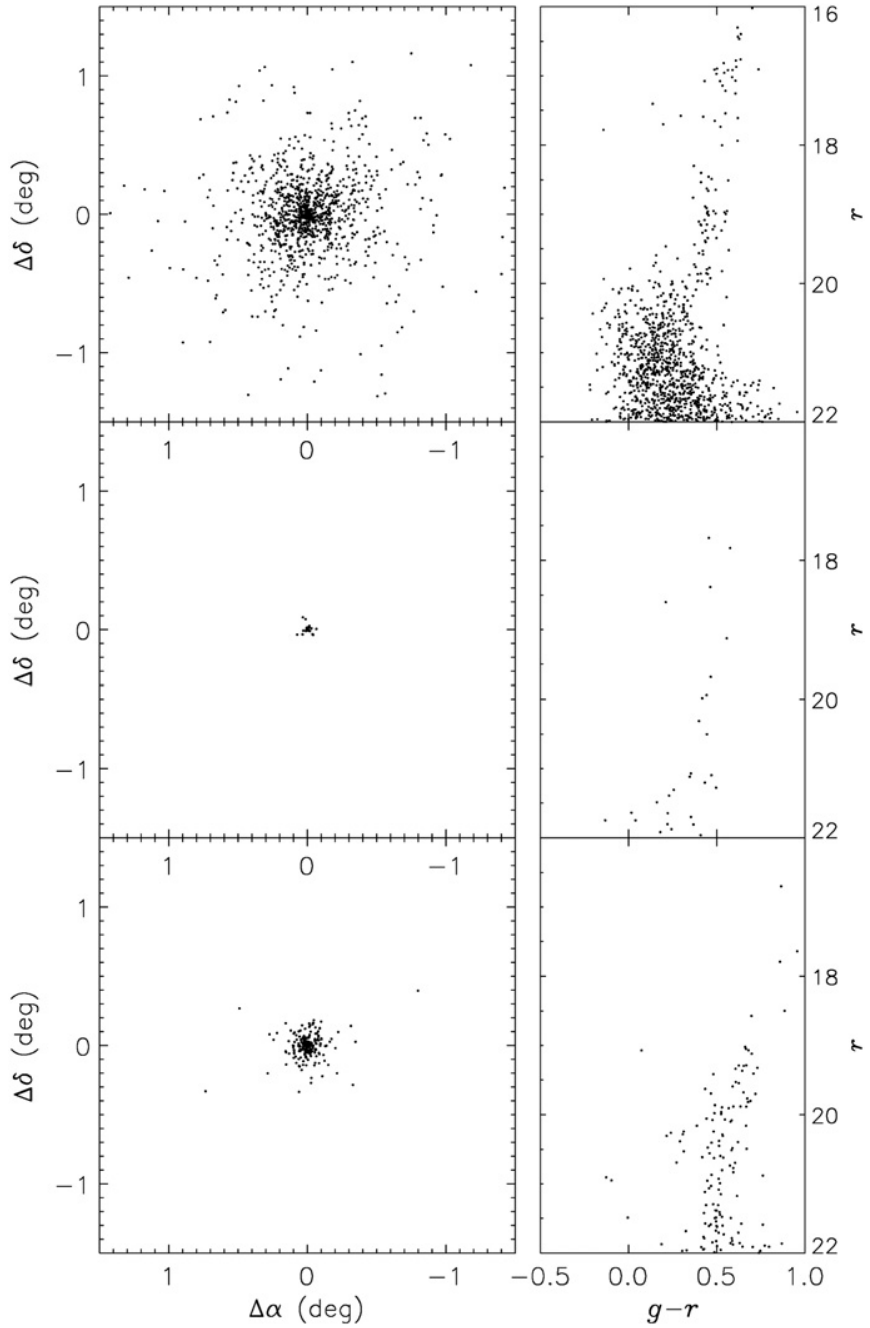

Figure 14. Simulated dSph systems. Top: $d=25 \mathrm{kpc}, r_{h}=250 \mathrm{pc}, M_{V}=$ -4.7 . Middle: $d=45 \mathrm{kpc}, r_{h}=40 \mathrm{pc}, M_{V}=-2.3$. Bottom: $d=100 \mathrm{kpc}$, $r_{h}=250 \mathrm{pc}, M_{V}=-5.6$. 


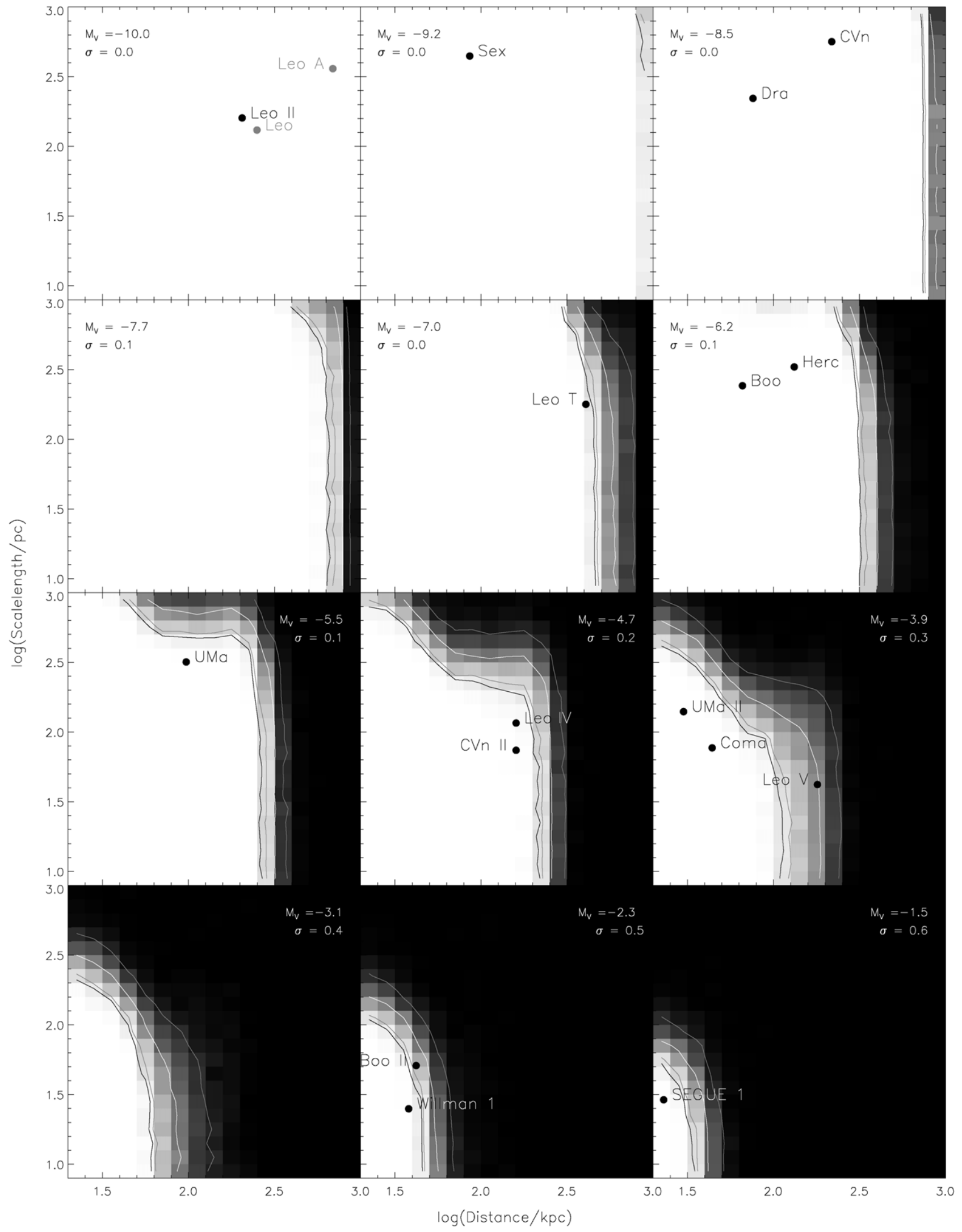

Figure 15. Detection efficiency for specific galaxy parameters. Each panel shows the detection efficiency as a function of distance and scale length for a particular number of galaxy stars. The average total absolute magnitude for each of these sets of galaxies is shown along with the standard deviation in magnitudes. Contours show the $90 \%, 84.13 \%, 50 \%$, and $15.86 \%$ levels. Sizes and distances of known MW dSphs are shown in the best-matching magnitude panel. Leo and Leo A are shown in gray as they are significantly brighter than $M_{V}=-10.0$. Values for newly discovered objects plus Draco are taken from Martin et al. (2008) except for Leo V (Belokurov et al. 2008). Values for Leo, Leo II, Leo A, and Sextans are taken from Mateo (1998). 
simulate systems at latitudes of $31^{\circ}, 53^{\circ}$, and $73^{\circ}$ and with $2^{x} \times 100$ stars brighter than $M_{V}=6$, where $x$ is an integer between 0 and 11 (giving a range of 100-204,800 stars). These stellar totals correspond to mean total magnitudes of $M_{V}=-1.5,-2.3,-3.1,-3.9,-4.7,-5.5,-6.2,-7.0$, $-7.7,-8.5,-9.2$, and -10.0 . For each of the 36 combinations of latitude and magnitude, we simulate a large number of galaxies with distances and physical scale lengths randomly generated with the limits $1.3<\log d / \mathrm{kpc}<3.0$ and $0.9<$ $\log r_{h} / \mathrm{pc}<3.0$. For the brightest and faintest systems we tailor these ranges to avoid redundant iterations; there is little to be gained by simulating an $M_{V}=-1.5$ system at $200 \mathrm{kpc}$, or an $M_{V}=-10.0$ system at $20 \mathrm{kpc}$. Hence, the total number of simulations for each magnitude/latitude combination varies, but is chosen such that there are typically 500 simulations in each $0.1 \log (d) \times 0.1 \log \left(r_{h}\right)$ bin of Figure 15 .

\section{DISSECTING EFFICIENCY TRENDS}

Figure 15 shows the detection efficiency of simulated dwarf galaxies as a function of luminosity, scale length, and distance at the median SDSS Galactic latitude $\left(53^{\circ}\right)$. Each of the MW satellites detected in SDSS are overplotted, not including Koposov 1 and 2. Each panel contains a gray-scale map of the detection efficiency for simulated galaxies of the mean absolute magnitude specified in the panel. Because the total magnitude varies for systems with a constant number of stars, we quote both the mean magnitude and the standard deviation of magnitudes for each panel. White shows regions of $100 \%$ efficiency, while black shows $0 \%$. The four contours, moving outward from $100 \%$ efficiency, show the $90 \%, 84.13 \%, 50 \%$, and $15.86 \%$ levels. The $84.13 \%$ and $15.86 \%$ levels were chosen to illustrate the $\pm 1 \sigma$ in detectability as a function of distance and size.

The greater the number of stars in a simulated galaxy, the less its absolute magnitude will vary between realizations, so the standard deviation in the integrated magnitudes of simulated galaxies contributing to each panel decreases with increasing luminosity. The $M_{V}=-7.7$ panel, which shows a small increase in standard deviation, marks a change in the way the galaxies are simulated; we are now simulating systems with more stars than are in our HST catalog so the simulated stars no longer have unique photometry drawn from this catalog. This amplifies the small number effect of single stars on the total magnitude for simulated galaxies with $M_{V}=-7.7$ and brighter.

Figure 15 illustrates the necessity for a large number of simulations as there are subtle features that would otherwise be unresolved. For example, this figure shows that the detectability of dwarfs is not a step function in distance, but rather slowly falls off at a rate that differs for systems with different total luminosities. This is in contrast to Koposov et al. (2008), who found a steep boundary between $100 \%$ and $0 \%$ efficiency. The gradual fall-off in dwarf detectability with distance will be discussed in more detail in Section 6.1, but we postpone detailed comparison with Koposov et al. (2008) until Section 7.

The critical factor affecting the detectability of an object with our algorithm is the number of stars brighter than $r=22.0$ that fall under the $r_{h}=4.5$ Plummer smoothing kernel. In the following sections, we use this to gain physical understanding of the features of Figure 15 and to derive an analytic expression to describe detection efficiency as a function of galaxy magnitude, size, distance, and Galactic latitude. This analytic expression, as well as a routine to interpolate detectability directly from the simulations, will be made publicly available and can be used, for example, to correct the MW satellite luminosity function as previously done by Koposov et al. (2008) or to make an estimate of the corrected radial distribution of MW satellites. Such endeavors are beyond the scope of this paper, but we use the function to estimate the total number of MW satellites that remain undetected, presented in Section 8.

\subsection{Efficiency Versus Distance}

Figure 15 shows that the detectability of resolved dwarfs around the MW is not a step function in distance. As distance to a dwarf galaxy increases, the number of stars brighter than $r=22.0\left(N_{r<22}\right)$ decreases. In an idealized scenario, the detectability of that dwarf would drop from $100 \%$ to $0 \%$ at a distance beyond which the number of resolved stars required to produce a detection is larger than $N_{r<22}$. As we have discussed in previous sections, random variations in the stellar luminosity function can be substantial in the faintest systems, hence $N_{r<22}$ will be affected by stochastic fluctuations. Moreover, the wide range in foreground densities at a given Galactic latitude (see Figure 12) impacts the detectability of two identical dwarfs. Therefore the transition from 1.0 to 0.0 detection efficiency is not expected to be a step function, but rather described by a Gaussian integral. Koposov et al. (2008) also modeled the detectability transition with a Gaussian integral, despite finding a steep decline. So detection efficiency as a function of $\log ($ distance $/ \mathrm{kpc})$, which for brevity we denote ${ }_{L} d$ can be described as

$$
\operatorname{DE}\left({ }_{L} d\right)=\operatorname{erf}\left(\frac{{ }_{L} \bar{d}-{ }_{L} d}{\sigma_{L} d}\right)
$$

where ${ }_{L} d$ is the logarithm of distance, and ${ }_{L}^{-} d$ and $\sigma_{L d}$ are the mean and standard deviation of $\log ($ distance $)$. The mean corresponds to the distance at which a system would be detected with $50 \%$ efficiency. The error function erf is defined as:

$$
\operatorname{erf}(x)=\frac{2}{\sqrt{\pi}} \int_{0}^{x} e^{-t^{2}} d t .
$$

Examination of Figure 15 shows that $\sigma_{L}$ (how quickly efficiency transitions from unity to zero) changes depending on luminosity. We would naively expect $\sigma_{L} d$ to continue increasing with decreasing brightness as small number statistics becomes more dominant. Instead it shows a maximum at $M_{V} \approx-3.9$ before decreasing. This is a result of the stochastic fluctuations and the derivative of the luminosity function; since $N_{r<22}$ varies for systems with the same total number of stars, the individual distance that each of these systems could be detected at also varies. As the number of stars above the brightness limit is dependent on the LF, the slope of the LF determines how $N_{r<22}$ changes with distance. Hence $\sigma_{L d}$ is smaller for the faintest objects when the MSTO is required for a detection because the $\mathrm{LF}$ is at its steepest at the turnoff.

\subsection{Efficiency Versus Scale length}

The fraction of a dwarf's stars within our 4'.5 spatial smoothing kernel decreases with increasing physical scale length and/ or decreasing distance. A system of some luminosity and distance that is detectable when its angular size is $\lesssim 4.5$ may thus be undetectable if those same stars are spread over a larger angular scale. As the concentration of stars increases we would expect detection efficiency to also increase. However, when the angular size of a dwarf is comparable with the smoothing kernel 


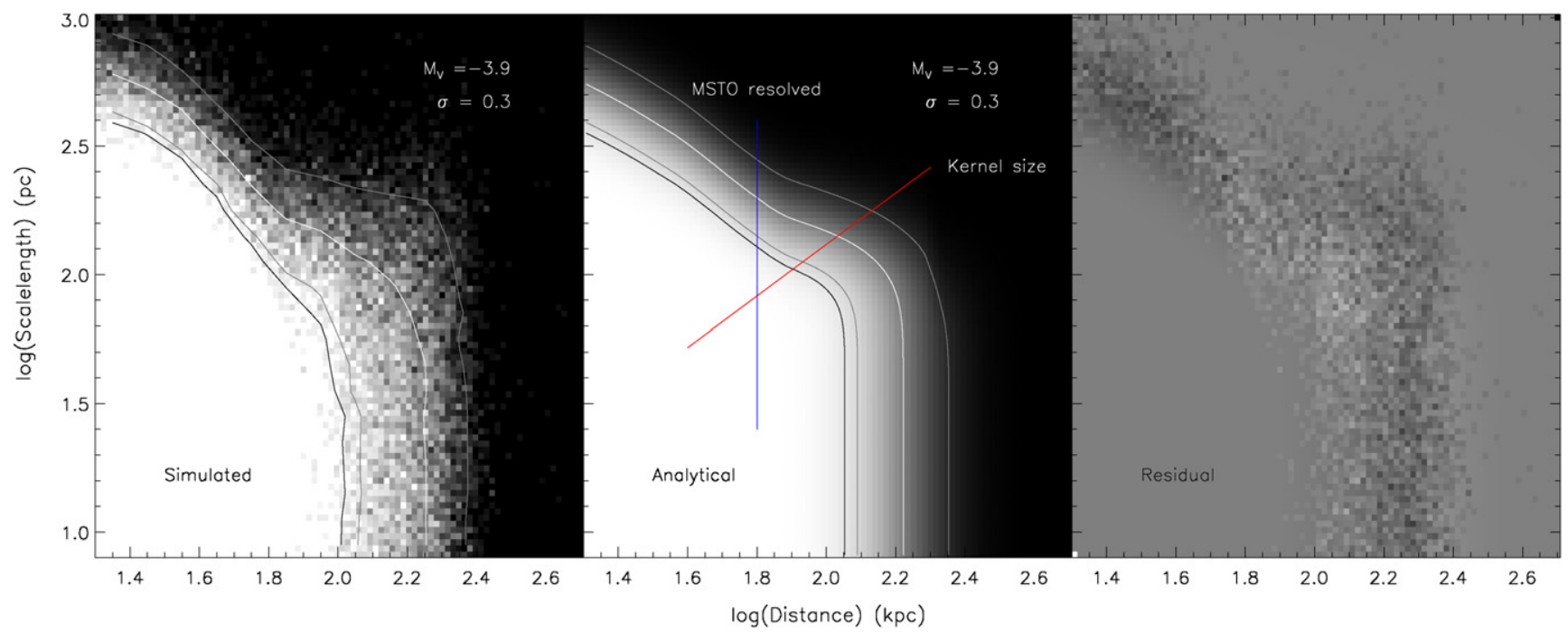

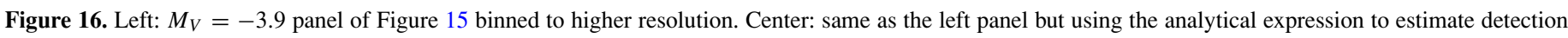
efficiency. Right: residual of the model-analytical efficiency.

(A color version of this figure is available in the online journal.)

size, the detectability does not appreciably improve with further decrease in size since the number of stars within the kernel is not significantly changing. Hence objects of this angular size or smaller will be detected with the same efficiency.

Once the angular size becomes larger than the kernel size, the number of stars within the kernel declines. The relationship between size and detection distance is dependent on the stellar luminosity function of the system. Take for example an object with an angular size larger than the smoothing kernel, detected with $50 \%$ efficiency at some distance. To keep the object at $50 \%$ efficiency as we continue to increase the physical size, the drop in efficiency can be counteracted by decreasing the object's distance. As this object is moved closer the number of stars above $r=22.0$ increases at a rate corresponding to the LF. At the distance when the MSTO becomes brighter than $r=22.0(\sim 65 \mathrm{kpc})$, the rapid increase in the number of stars corresponds to a sudden improvement in detection efficiency, evident in Figure 15 at $\log (d) \approx 1.8$ in the $M_{V}=-5.5,-4.7$, and -3.9 panels. As with distance, efficiency versus scale length can be modeled by a Gaussian integral, but with the mean and standard deviations as functions of distance; so

$$
\operatorname{DE}\left({ }_{L} r_{h}\right)=\operatorname{erf}\left(\frac{{ }_{L^{-}}{ }_{h}\left({ }_{L} \bar{d}\right)-{ }_{L} r_{h}}{\sigma_{L} r_{h}\left({ }_{L} d\right)}\right) .
$$

\subsection{Analytically Expressing Detection Efficiency}

Combining the previous two results, we can analytically describe detectability with a Gaussian integral over $\log d$ multiplied by another Gaussian integral over $\log r_{h}$. To introduce magnitude $M_{V}$ and latitude $b$, we set the means $\left({ }_{L} \bar{d},{ }_{L} r_{h}\right)$ and standard deviations $\left(\sigma_{L d}, \sigma_{L} r_{h}\right)$ in the integrals to be functions of $M_{V}$ and $b$. Therefore the detection efficiency can be expressed as

$\mathrm{DE}=\operatorname{erf}\left(\frac{{ }_{L} \bar{d}\left(M_{V}, b\right)-{ }_{L} d}{\sigma_{L} d\left(M_{V}, b\right)}\right) \times \operatorname{erf}\left(\frac{{ }_{L^{2}}{ }_{h}\left({ }_{L} d\left(M_{V}, b\right)\right)-{ }_{L} r_{h}}{\sigma_{L r_{h}}\left({ }_{L} d\left(M_{V}, b\right)\right)}\right)$

The means and standard deviations can be found by fitting Gaussian integrals along the distance and scale length axes of the panels in Figure 15. The main source of uncertainty in this expression is the galaxy luminosity, which, while correlated
Table 3

Comparison of Interpolated and Analytical Detection Efficiencies

\begin{tabular}{lccc}
\hline \hline Object & Interpolated & Analytical & Difference \\
\hline Boo & 99.84 & 99.98 & -0.14 \\
Boo II & 90.39 & 95.96 & -5.57 \\
CVn & 100.0 & 100.0 & 0.0 \\
CVn II & 98.26 & 99.07 & -0.81 \\
Com & 99.71 & 100.0 & -0.29 \\
Dra & 100.0 & 100.0 & 0.0 \\
Her & 99.82 & 99.83 & -0.01 \\
Leo & 100.0 & 100.0 & 0.0 \\
Leo II & 100.0 & 100.0 & 0.0 \\
Leo A & 100.0 & 100.0 & 0.0 \\
Leo IV & 98.65 & 99.77 & -1.12 \\
Leo V & 83.56 & 91.27 & -7.71 \\
Leo T & 93.41 & 99.38 & -5.97 \\
Segue 1 & 100.0 & 100.0 & 0.0 \\
Sex & 100.0 & 100.0 & 0.0 \\
UMa & 99.97 & 99.86 & 0.11 \\
UMa II & 100.0 & 99.96 & 0.04 \\
Will 1 & 98.56 & 99.30 & -0.74 \\
\hline
\end{tabular}

with the number of stars, can vary by over a magnitude for systems of equal detectability. The function does however give a good statistical approximation from which to estimate the properties of the true MW satellite population. In Figure 16, we compare the $M_{V}=-3.9$ panel of Figure 15 with the analytical function. There is good agreement between the empirical and analytical detection efficiencies with a $1 \sigma$ deviation of only $\sim 8.7 \%$ across the entire range of parameters. For reference the size of the 4.5 smoothing kernel and the distance at which the MSTO becomes resolved are shown in the center panel in red and blue, respectively.

The analytical efficiency is compared in Table 3 with the interpolated efficiency from the grids in Figure 15 for real MW dwarfs. For all objects besides Boötes II, Leo V, and Leo T, the difference is within $\sim 1 \%$. At first inspection, it may seem odd that all objects have a very high, almost $100 \%$, efficiency, but given that most of the parameter space probed by our simulations yields either zero or unity efficiency, it is not unexpected that the handful of objects detected in this vast volume are detected with high efficiency. 


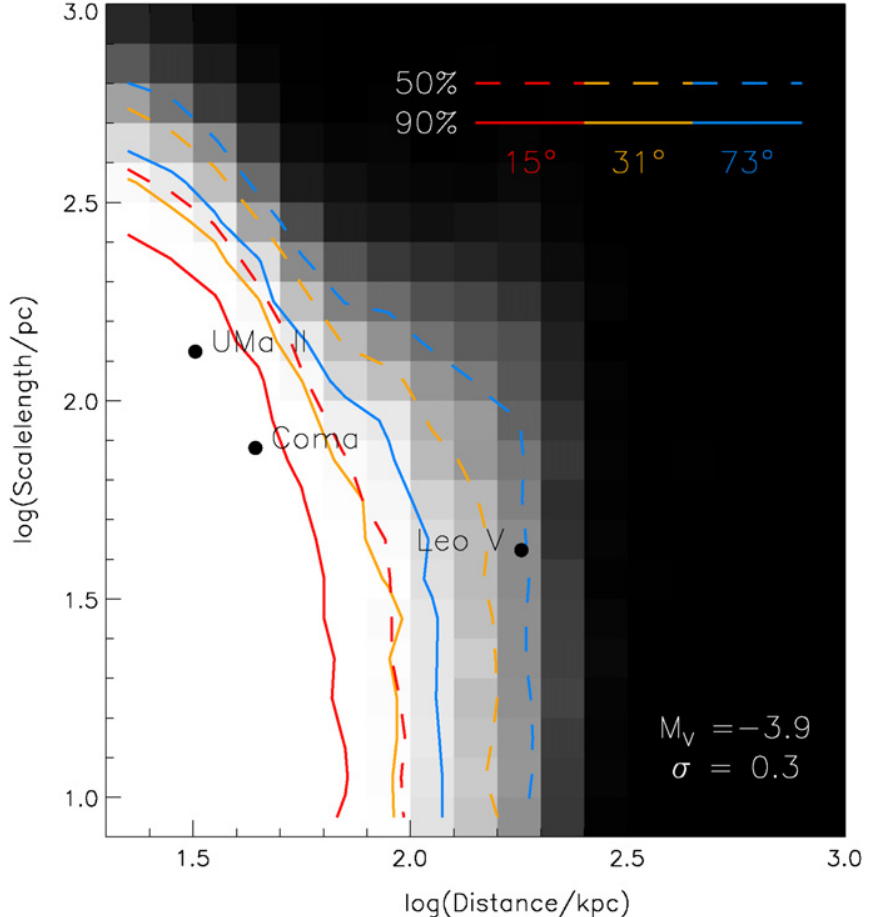

Figure 17. The same as the $M_{V}=-3.9$ panel of Figure 15, but showing the $50 \%$ and $90 \%$ contours of the simulations at $b=73^{\circ}$ (blue), $31^{\circ}$ (orange), and $\sim 15^{\circ}$ (red).

\subsection{Efficiency Versus Latitude}

Unlike the other parameters which vary a dSph's signal strength, Galactic latitude affects detection efficiency by changing the foreground density, and therefore noise above which we must detect a signal. If latitude plays a significant role in the detectability of dwarfs, then it must be taken into account when making any corrections to the MW satellite census. Figure 17 shows the $M_{V} \approx-3.9$ panel of Figure $15\left(b=53^{\circ}\right)$ with the addition of the 50\% detection efficiency (dashed) and $90 \%$ detection efficiency (dotted) contours of the $b=31^{\circ}$ (orange) and $73^{\circ}$ (blue) simulations overplotted. As expected, an object of given size and luminosity will not be detectable as far away at low latitudes as it would be closer to the Galactic pole. For example, an object with $r_{h} \approx 30 \mathrm{pc}$ and $M_{V} \approx-3.9$ at $b=73^{\circ}$ can be detected with $90 \%$ efficiency as far as $\sim 120 \mathrm{kpc}$, while the same object at $b=31^{\circ}$ has a $90 \%$ efficiency at $\sim 95 \mathrm{kpc}$.

To anticipate the effect that varying Galactic foreground will affect future dwarf searches in data that go closer to the Galactic plane than SDSS, we also repeat the simulation of $M_{V} \approx-3.9$ galaxies at a foreground density of 10,000 stars per $\mathrm{deg}^{2}$, approximating a latitude of $\sim 15^{\circ}$. The $50 \%$ and $90 \%$ detection efficiency contours (red) for these simulations are also shown in Figure 17. This further reduces the $90 \%$ detection distance of our example object to $\sim 80 \mathrm{kpc}$ but demonstrates that future surveys should still detect dwarfs at relatively low Galactic latitudes, barring extinction effects.

To check that latitude has the same lack of effect over different magnitudes, we calculate the $50 \%, 90 \%$, and $99 \%$ detection efficiency distances for a $r_{h}=100 \mathrm{pc}$ object over the magnitude range $-1.5>M_{V}>-7.0$ at $b=31^{\circ}, 53^{\circ}$, and $73^{\circ}$ (Figure 18 , note that in this figure the distance scale is linear). The $53^{\circ}$ and $73^{\circ}$ curves are indistinguishable and the $b=31^{\circ}$ curve is typically less than $\sim 20 \mathrm{kpc}$ lower. This demonstrates that over the DR6 footprint, latitude does not play an important

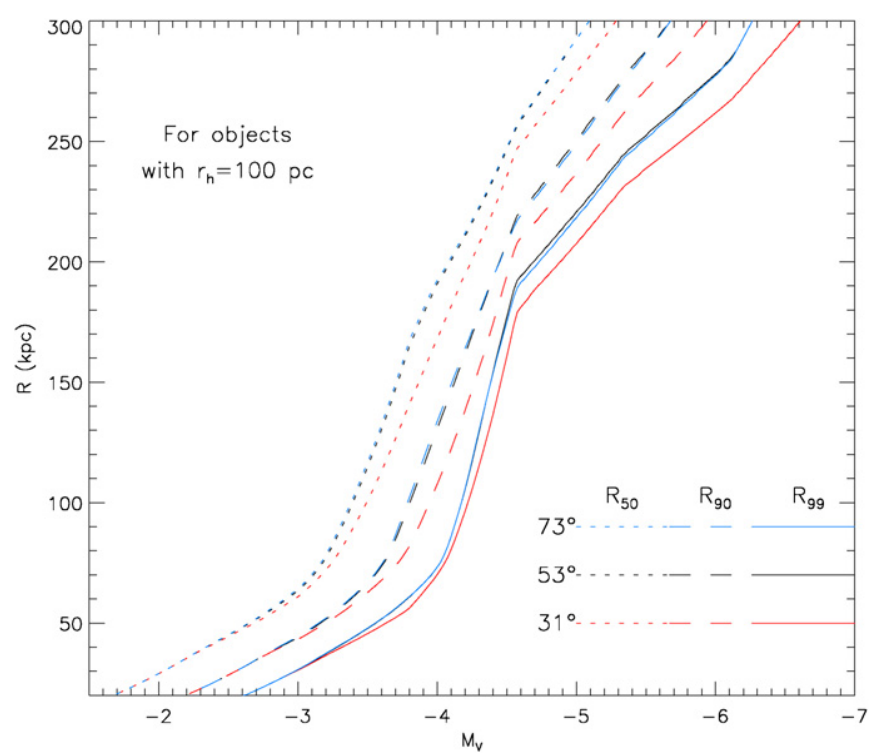

Figure 18. 50\%, 90\%, and 99\% completeness distances for a $r_{h}=100 \mathrm{pc}$ object as a function of magnitude at three Galactic latitudes: $31^{\circ}, 53^{\circ}$, and $73^{\circ}$. The $53^{\circ}$ and $73^{\circ}$ curves are virtually indistinguishable showing that latitude does not significantly impact satellite detection over the latitude range of DR6.

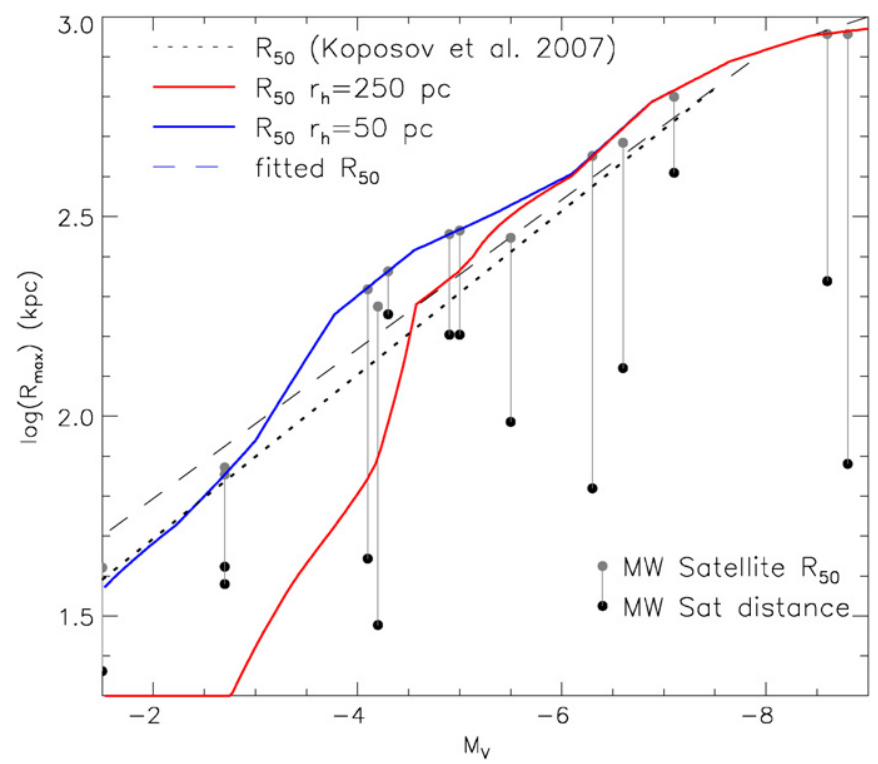

Figure 19. Comparison of the $50 \%$ detection distance as a function of magnitude for K08 (dotted) and for our analytical efficiency using $r_{h}=250 \mathrm{pc}$ (red) and $r_{h}=50 \mathrm{pc}$ (blue). MW dwarfs are shown as filled circles.

role on average in the detectability of objects. However, if we are unlucky, then individual objects could by chance lie in the directions of unusually high foreground counts.

\subsection{Comparing $R_{99}, R_{90}$, and $R_{50}$}

We use the analytical expression derived in Section 6.3 to estimate the distance at which each of the MW dwarfs would be detected with $50 \%$ efficiency, $R_{50}$ (Figure 19, gray dots). Because $R_{50}$ depends on $r_{h}$, as well as luminosity, we also for reference show $R_{50}$ for objects with $r_{h}=250 \mathrm{pc}$ (red) and $r_{h}=50 \mathrm{pc}$ (blue). These lines show that the detectability of the lowest luminosity dwarfs is severely reduced for large scale sizes. Objects with Segue 1-, Boötes II-, or Willman 1-like luminosities would not have been detectable with scale sizes of $100 \mathrm{pc}$ or larger, even at very nearby distances. This size 
bias is important to bear in mind, particularly given that the three M31 satellites discovered by McConnachie et al. (2008) highlight regions of dwarf galaxy parameter space that have not previously been observed.

Assuming the size-luminosity distribution of known satellites is representative of all satellites we can ignore size and approximate $R_{50}$ as a function of $M_{V}$ by linear fit to the $R_{50}$ of actual dwarfs:

$$
\log R_{50}=-0.187 M_{V}+1.420 \text {. }
$$

For comparison, the Koposov et al. (2008) equivalent of $R_{50}$ is shown in Figure 19 (dotted line) obtained from Table 3 in their paper, and discussed further in Section 7. For reference, the actual distances to the MW dwarfs are shown as black dots.

A more useful quantity might be $R_{\text {complete, the maximum }}$ distance at which objects can be detected. Although we choose to use "complete" to refer to $90 \%$ detectability, complete could be defined as, say $90 \%$ or $99 \%$ efficiency. This can also be approximated by a linear fit to the results for the actual MW dwarfs:

$$
\log R_{90}=-0.204 M_{V}+1.164
$$

or

$$
\log R_{99}=-0.217 M_{V}+1.005 \text {. }
$$

These relationships again assume that the known satellites are typical of the MW satellite population as a whole, i.e., objects with luminosities comparable with Segue 1 are similar in size to Segue 1 and not significantly larger.

Returning to Figure 18 we see the distance range over which detectability changes from $99 \%$ to $50 \%$. $R_{90}$ is typically $\sim 20 \mathrm{kpc}$ closer than $R_{50}$, and $R_{99}$ is $\sim 20 \mathrm{kpc}$ closer still. From this figure we can also see that objects brighter than $M_{V} \approx-6.5\left(M_{V} \approx\right.$ $-5.9)$ are detected with $99 \%(90 \%)$ efficiency out to $300 \mathrm{kpc}$. We can infer from this that all dwarfs within the MW virial radius brighter than $M_{V} \approx-6.5$ are known, and any satellites still undetected are likely to be comparable with objects such as Coma Berenices, Boötes II, or Segue 1, at distances greater than $\sim 40 \mathrm{kpc}$. An ultrafaint satellite such as Segue 1 can only be detected with $50 \%$ efficiency out to $\sim 40 \mathrm{kpc}$; there may be many more such objects beyond this distance.

The code for interpolating detection efficiency from our simulations as well as the analytical function will be made available for download at the Astronomical Journal Web site. Interpolation will give more accurate results, but the analytical function will provide flexibility for customization to suit individual needs and implementation of any future improvements.

\subsection{Caveats}

An underlying assumption of our simulations is that the DR6 point-source catalog is consistently $100 \%$ complete to $r=22.0$. This assumption may result in optimistic detection efficiency estimates for the faintest and furthest systems. These faintest and more distant systems would also be subject to the human element; a real object may be detected by the algorithm but on visual inspection be disregarded as background galaxy cluster or other contaminant. Finally, sources in our simulated dSphs are distributed circularly symmetrically. Martin et al. (2008) find that the ultrafaint satellites are in fact quite elliptical, which due to our circular Plummer smoothing kernel, possibly results in overestimated efficiencies for objects such as Hercules, UMa, and UMa II with ellipticities of $0.68,0.80$, and 0.63 , respectively.

\section{COMPARISON WITH KOPOSOV ET AL. (2008)}

Besides Willman et al. (2002) from which this work follows, two other surveys have recently uniformly searched SDSS for MW satellites, namely Liu et al. (2008) and Koposov et al. (2008), henceforth K08. Liu et al. (2008) conducted a straightforward search and presented five satellite candidates. Koposov et al. (2008) present a study comparable with this work, and follows from the "Field of Streams" (Belokurov et al. 2006a) that led to the discoveries of nine of the new MW satellites. Here we compare our work in detail with Koposov et al. (2008) and summarize the main differences in Table 4.

The aim of Koposov et al. (2008) was to present a luminosity function of the MW satellites corrected for a luminosity bias. Their analysis discovered two new extremely faint globular clusters, Koposov 1 and 2 (Koposov et al. 2007). In principle, our analysis is quite similar to K08 in that they apply a color cut, smooth the stellar counts, and look for statistically significant overdensities. There are several distinctions however that we detail below.

K08 employed a $g-r<1.2$ color cut to remove a substantial fraction of MW foreground stars and a $r<22.5$ cut to limit the influence of background galaxies and increasing uncertainties/ incompleteness. Our color-magnitude cuts are tailored to old stellar populations at 16 different distances which serve to eliminate more foreground stars than the looser K08 cut. The looser K08 cut leaves enough stars that a complicated set of detection thresholds is unnecessary, whereas we must consider the effects of non-Gaussianity in low densities (see Section 3.4). K08 deals with background galaxy clusters, a major source of contaminant overdensities, by producing a galaxy clustering significance in the same manner as the stellar clustering; anywhere that a stellar overdensity occurs without a corresponding galaxy overdensity is much more likely to be a true stellar overdensity. Our algorithm only includes stars as faint as $r=22.0$ and as such we have fewer galaxy cluster contaminant detections.

The most substantial difference between our work and K08 is how we derive the detection limits of our algorithms. Like our work, K08 simulated artificial galaxies to explore the detection efficiency as a function of size, distance, and luminosity. K08 simulated 8000 galaxies over a similar range of parameters as our study, but with only $\sim$ eight objects per $0.3 \log (d) \times$ $0.3 \log \left(r_{h}\right) \times 0.8 \mathrm{mag}$ bin. There is considerable noise evident in the detection limits (their Figure 6), and all of the new satellites appear to lie on the edge of detectability. K08 observed a steep, but finite, transition from unity to zero detection efficiency which they attributed to the large range of distances that fall within each size-luminosity bin. However, as discussed in our Section 5.2, a large number of simulated galaxies is essential for each permutation of dwarf galaxy parameters to effectively map their detectability. Our high-resolution detection maps $\left(\sim 500\right.$ objects per $0.1 \log (d) \times 0.1 \log \left(r_{h}\right) \times$ 0.8 mag bin, $\times 3$ latitudes) show that the detectability of a dwarf drops off slowly with size and distance, and that only Leo T, Leo IV, Leo V, Boötes II, and Willman 1 lie close to the edge of detectability. The difference between $90 \%$ and $10 \%$ efficiency typically occurs over 0.2 dex in distance $(\mathrm{kpc})$ and 0.3 dex in size (pc; see Figure 15).

Both the K08 and our detection limit calculations suffer from the implicit assumptions that the SDSS point-source catalog is complete to the photometry limit and that dwarfs are circularly symmetric. These two assumptions yield detec- 
tion limits that may be optimistic. The K08 study includes stars to a limiting magnitude of $r=22.5$, a half-magnitude fainter than our limit of $r=22.0$. We thus expect that the completeness assumption may impact their calculated limits more than ours.

To directly compare the effectiveness of both algorithms we return to Figure 19, showing the distance at which an object is detected with $50 \%$ efficiency, $R_{50}$, as a function of magnitude. The dotted line shows the K08 $R_{50}$ which was determined by fitting a limiting magnitude and surface brightness to the seven distance panels in their Figure 10. The gray dots show our $R_{50}$ derived from the analytical efficiency function for each of the MW dwarfs in DR6, and the dashed line shows the best linear fit to these points. The red and blue curves show $R_{50}$ calculated for objects of $r_{h}=250 \mathrm{pc}$ and $r_{h}=50 \mathrm{pc}$, respectively.

Although this comparison indicates that we have comparable limits, our calculated detection efficiencies of each dwarf are all greater than $90 \%$ while Table 2 of K08 lists efficiencies as low as $47 \%$ (neglecting Boötes II). While Boötes II is not detected with the standard algorithm of K08, it is a comparatively strong detection in our algorithm. We note that the tabulated K08 efficiencies for the known MW dwarfs appear inconsistent with their fitted $R_{50}$, which places some dwarfs much closer than $R_{50}$ than their actual efficiencies would indicate. The increased dwarf detectability of our survey is owing to a combination of different techniques and our less stringent detection threshold. We set our thresholds to strictly eliminate truly random false positives expected while still yielding new candidates, and hence have $\sim 30$ unknown detections above our thresholds. Although upon visual inspection of their CMDs many of these detections appear unlikely to be new dSph satellites, they may also be tidal debris or distant galaxy clusters. However, K08 set their detection thresholds just loose enough to retain all known objects; UMa is their weakest detection and there are only three unknown detections above this threshold.

\section{THE STILL MISSING SATELLITES}

A substantial driving force of this work is the missing satellite problem, which the discovery of so many new objects in the span of three years has shown is far from being observationally exhausted. There are still large regions of parameter space where objects are undetectable, so there can easily exist more objects within the DR6 coverage that remain hidden. Future surveys such as the Stromlo Missing Satellites (SMS) Survey and Pan-STARRS may be able to detect some of these objects, and we can use our model of detectability to estimate how many there may be.

We use a simplified version of the approach used in Tollerud et al. (2008). We first assume that the radial distribution of dwarf galaxies matches that of all well resolved subhalos of the Via Lactea simulation (Diemand et al. 2007). Tollerud et al. (2008) discusses this assumption in detail; we realize this may not reflect the true MW dwarf distribution, but our qualitative results are fairly robust to the assumed profile. For each satellite detected in DR6, we then determine $R_{90}$ (or $R_{99}$ ), the maximum distance to which a satellite of similar properties would be detected with $90 \%$ (99\%) efficiency. For each value of $R_{90}\left(R_{99}\right)$, we determine from the Via Lactea subhalo radial profile what fraction of satellites should be within this distance, and weight each satellite accordingly.

Using all DM subhalos with more than 1000 particles within $r_{\mathrm{vir}}=289 \mathrm{kpc}$, and adopting a MW virial radius of $258 \mathrm{kpc}$
(Klypin et al. 2002), we estimate $\sim 13(\sim 24)$ satellites within the MW virial radius in the DR6 footprint. Twelve of these would be the known objects Boötes, Draco, Canes Venatici I and II, Coma Berenices, Leo I, Leo II, Leo IV, Leo V, Hercules, Ursa Major, and Ursa Major II, leaving one (12) possible missing satellite(s). From our simulations, objects brighter than $M_{V}=-6.5$ are detectable with $>99 \%$ efficiency out to the virial radius, so we would expect that a relatively small number of the faintest systems are missing. These missing satellites may be amongst our candidates, or be either like Coma Berenices or fainter objects in the outer halo. Whether or not future searches reveal such objects may validate the assumed radial distribution. If we assume an isotropic sky distribution of satellites, $\sim 13(\sim 24)$ objects within DR6 equates to $\sim 52(\sim 96)$ across the whole sky.

If we include the ambiguous objects Segue 1, Willman 1, and Boötes II in the calculation, then the $R_{90}\left(R_{99}\right)$ DR6 estimate would be $\sim 56(\sim 85)$ satellites only 15 of which are known, or $\sim 224(\sim 340)$ across the sky. The ambiguity of Segue 1, Willman 1, and Boötes II has considerable effect on the extrapolated MW census, underscoring the need for an understanding of these extremely faint systems.

These estimates assume that the sizes and luminosities of the known satellites in DR6 are representative of the MW satellite population as a whole. Based on our detection limits we cannot make any statements regarding extremely diffuse, low luminosity systems that are undetectable by SDSS. The results also depend on the radial distribution assumed. If we instead assume that the MW's dwarf population follows the radial distribution of the MW dSphs known prior to 2004, then our $R_{90}$ inferred total number of dwarfs (with size-luminosities similar to those known) within DR6 is 12 , or 25 including Segue 1, Willman 1, and Boötes II. This implies that all or most satellites within DR6 would be known.

Tollerud et al. (2008) use the detection limits of Koposov et al. (2008) to similarly estimate the true number of satellites within DR5 for a number of scenarios. The most comparable scenario to our assumptions (a limiting distance of $300 \mathrm{kpc}$, including all satellites except Segue 1 which is not in DR5) gives a result of $322_{-75}^{+144}$ satellites, consistent with our results of 232 for $R_{90}$ and 344 for $R_{99}$.

\section{ALLOWING A LITTLE LATITUDE}

Substantial effort has gone into the observation and interpretation of the spatial distribution of the satellites of disk galaxies, in particular that of the MW satellites. However, there is neither agreement on whether the MW satellites have a truly anisotropic spatial distribution, nor whether we expect them to. Pre-SDSS, Kroupa et al. (2005) found that the distribution of known MW satellites could be described by a disk of finite width, aligned almost perpendicularly to the MW disk. This was in agreement with the "Holmberg" effect (Holmberg 1969), that the closest satellites to a host galaxy were observed to be preferentially aligned with the minor axis of the host. This disklike distribution seemed incompatible with $\Lambda$ CDM, but Kang et al. (2005) reasoned that if satellites follow the distribution of the host DM profile rather than that of the substructure then the dozen observed MW satellites could statistically lie in a disklike structure, although the orientation of this disk is arbitrary. Piatek et al. (2007) used proper motions derived from HST observations to show that this "Great Disk of MW Satellites" was not a persistent structure; the orbits of the dwarfs would not contain them within this disk. Metz et al. (2008) refute this conclusion, finding instead that the orbital poles of most MW satellites 
Table 4

Summary of Comparison with Koposov et al. (2008)

\begin{tabular}{|c|c|c|}
\hline & This work & Koposov et al. (2008) \\
\hline Survey area & DR6 - $9500 \mathrm{deg}^{2}$ & DR5 - $8000 \mathrm{deg}^{2}$ \\
\hline Source cuts & Isochrone template at 16 distance intervals & $g-r<1.2$ and $r<22.5$ \\
\hline Smoothing kernel & 4.5 Plummer profile & $\sigma=2^{\prime}, 4^{\prime}, 8^{\prime}-\sigma=60^{\prime}$ Gaussians \\
\hline Threshold & Multiple, function of foreground density & Fixed, considers background galaxies \\
\hline Modeled detection limits & HST obs of $3 \mathrm{MW}$ dSphs & M92 locus \\
\hline Number of simulations & $3,825,000$ & $\begin{array}{c}8000 \text { for general simulation + } \\
1000 \text { each for known dwarfs } \\
\text { within DR5 }\end{array}$ \\
\hline $\begin{array}{l}\text { Efficiency map bin size } \\
\left(\log \left(r_{h}\right) \times \log (d) \times M_{V}\right)\end{array}$ & $0.1 \times 0.1 \times 0.8$ & $0.3 \times 0.3 \times 0.8$ \\
\hline $\begin{array}{l}\text { Simulation density } \\
(\mathrm{n} \text { per } 0.3 \times 0.3 \times 0.8 \text { bin })\end{array}$ & $\sim 4500$ (×3 latitudes $)$ & $\sim 8$ \\
\hline
\end{tabular}

place them in a rotationally supported disk of satellites. Studies on the satellites of other galaxies from SDSS also yield conflicting results. Bailin et al. (2008) affirm the Holmberg effect while Brainerd (2005) find that satellites lie preferentially along the major, not minor, axis of the host. Zentner et al. (2005) reexamine the problem from a theoretical point of view, stating that DM substructure is not completely isotropic and that the MW satellite distribution can, albeit with a very low probability, be drawn from a DM subhalo distribution.

Within the standard $\Lambda \mathrm{CDM}$ structure formation scenario, satellite galaxies without DM could be formed in gas-rich tidal tails during vigorous early galaxy-galaxy interactions (Okazaki $\&$ Taniguchi 2000). Families of such tidal dwarfs would have correlated orbital angular momenta and may appear as disklike arrangements about some hosts. This would support the apparent disk of satellites (Kroupa et al. 2005; Metz et al. 2007) and its correlated orbital angular momenta (Metz et al. 2008). It is therefore crucial to further constrain the spatial and orbital angular momentum properties of the satellites to reveal their true nature which is intimately related to the formation of the MW.

A caveat of past studies of the MW dwarf distribution is that the sky had not been uniformly searched for satellites and the effect of Galactic latitude on the observability of dwarfs had not been thoroughly quantified. In their detailed study, Kleyna et al. (1997) showed that latitude strongly affected the detectability of MW satellites with their technique. Our uniform study of SDSS DR6 takes their approach a step further and provides a detailed quantitative description of dwarf detectability over the footprint of our survey. We established in Section 6.4 that the average detectability of the known satellites does not significantly vary over the DR6 footprint. We can thus compare the latitude and longitude distributions of the MW satellites within the DR6 footprint with that expected if they are randomly distributed. We perform a Kolmogorov-Smirnov $(\mathrm{K}-\mathrm{S})$ test to determine whether the satellites detected in DR6 show statistically significant spatial anisotropy. Figure 20 shows the cumulative distributions (black lines) of latitude (top) and longitude (bottom) by area of the DR6 footprint. Overplotted on both panels are the cumulative distributions of MW satellites, both ignoring the ambiguous objects Boötes II, Willman 1, and Segue 1 (blue) and including them (red). A K-S test on these distributions with the entire DR6 area yields probabilities of isotropic distribution of 0.16 over longitude and 0.72 over latitude, or 0.06 and 0.79 if we include Segue 1, Willman 1, and Boötes II. We also randomly pick 12 (or 15) points from DR6 coverage, weighted by area, and repeat the $\mathrm{K}-\mathrm{S}$
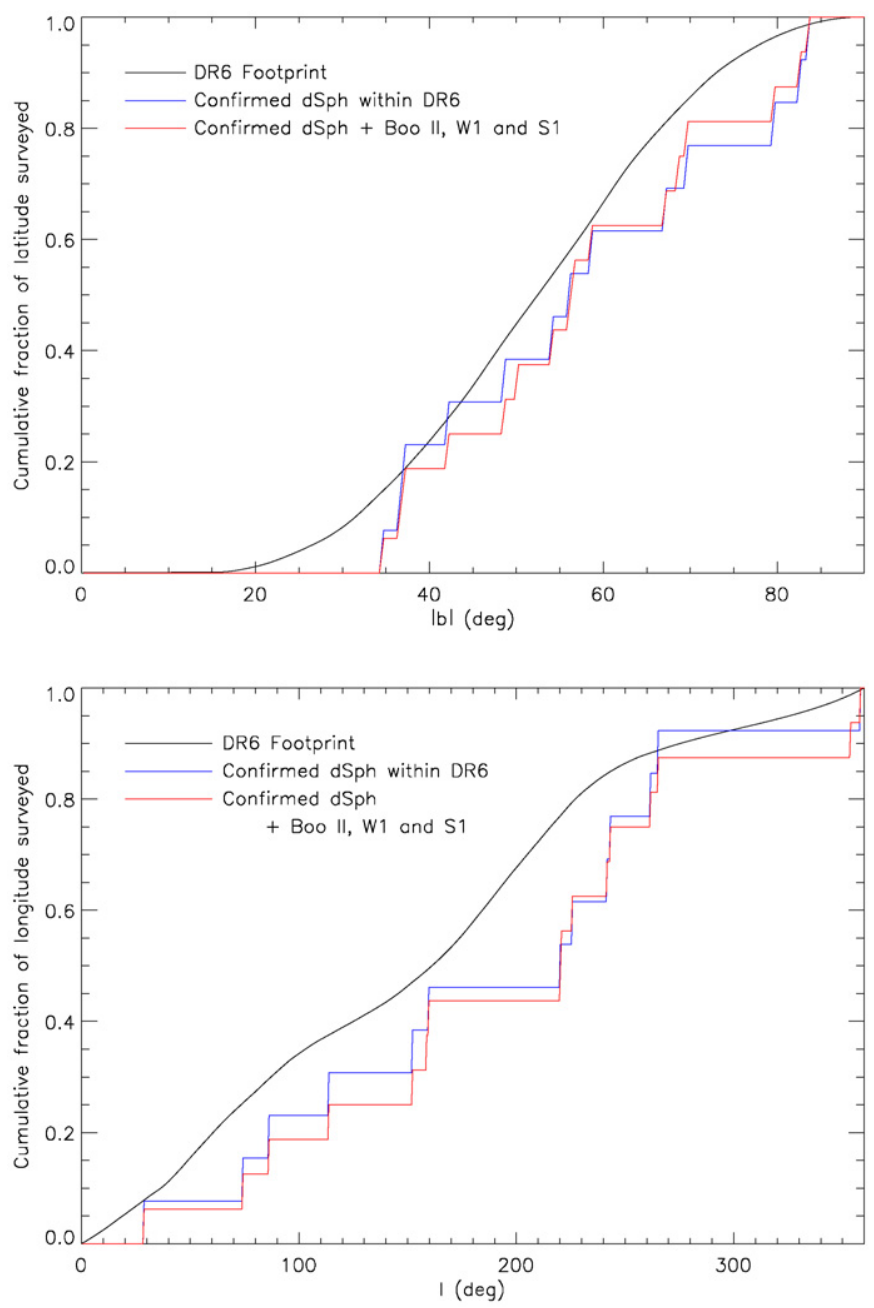

Figure 20. Top: cumulative histogram of the Galactic latitude of the DR6 footprint weighted by area (black). Blue shows the cumulative histogram of the latitudes of confirmed dSphs within DR6 and the MW virial radius (Boo, Dra, CVn, CVn II, Com, Leo I, Leo II, Leo IV, Her, UMa, and UMa II). Red shows the same but including Boötes II, Willman 1, and Segue 1. Bottom: same as the top panel, but for Galactic longitude.

(A color version of this figure is available in the online journal.)

test 1000 times. The mean resulting probabilities of isotropic distributions are $0.45 \pm 0.29$ over longitude and $0.62 \pm 0.27$ over latitude, or $0.34 \pm 0.28$ and $0.55 \pm 0.28$ with Segue 1 , Willman 1, and Boötes II. Hence, a conclusive result on 
the isotropy of the MW satellites awaits further data as our test shows that by considering DR6 alone either scenario is plausible.

\section{CONCLUSION}

The dwarf galaxy satellites of the MW provide excellent opportunities to further our understanding of galaxy formation and near-field cosmology. They can be resolved into individual stars allowing detailed studies of their structure, kinematics, and composition. They have also been cause for concern regarding their interpretation as the luminous components of DM substructure; it has been argued that the number and spatial distribution of these satellites are inconsistent with $\Lambda$ CDM structure formation scenarios. The commencement of the SDSS triggered a cascade of discoveries, with 14 new satellites discovered. The limited spatial coverage and photometric depth of SDSS suggests that many, if not most, MW satellites are still yet to be discovered. The coming years are likely to bring the MW satellite census toward completeness as new survey telescopes such as SkyMapper, Pan-STARRS, and LSST come online.

We present here the method used to search the SDSS DR6 for ultrafaint MW satellite galaxies. By screening for stars consistent with an old population at a fixed distance, we enhance the signal of a dSph over the MW foreground. Smoothing with a kernel corresponding to the expected surface density profile further elevates the $\mathrm{dSph}$ above the foreground, and our comprehensive thresholds account for varying stellar density and more diffuse objects.

Applying our algorithm to SDSS DR6, we recover the "classical" and recently discovered dSphs, as well as 17 globular clusters and two open clusters. We also have 30 unidentified detections, some of which may be new satellites. The discovery of Leo $\mathrm{V}$ demonstrates the difficulty in following-up dwarf candidates; while we detect Leo V, there are several unknown detections of greater significance that may prove to be something. However, observing these weakest candidates is a rather hit and miss affair, as pointed out by Belokurov et al. (2008).

To compare the known dwarf galaxy population of the MW with predictions, it is essential to have a very well-defined dwarf selection function. To do this, we thoroughly model the detection efficiency of systems covering a wide range in parameter space by simulating more than 3,000,000 galaxies. We fit various functions to the resulting detection efficiency contours to semianalytically describe efficiency as a function of magnitude, size, distance, and Galactic latitude. Using the results of our detailed investigation of dwarf detectability, we show that

1. Assuming a Via Lactea subhalo radial distribution and that $R_{\text {complete }}=R_{90}$, there should be $\sim 13$ satellites within DR6, 12 of which are known. If we include Segue 1, Willman 1, and Boötes II in this calculation, this estimate jumps to $\sim 56$, only 15 of which are known.

2. Dwarf detectability shows a smooth transition from $100 \%$ to $0 \%$ over size and distance. For example, the distance at which a CVn II-like object is detected with $90 \%$ efficiency is $200 \mathrm{kpc}$, compared with $316 \mathrm{kpc}$ for $10 \%$ efficiency.

3. Galactic latitude does not significantly impact the detection of satellites over the DR6 footprint, and surveys of similar quality should still detect dwarfs as low as $b \approx 15^{\circ}$. All of the satellites discovered in SDSS would have been detected at any latitude.
4. The census of MW satellites brighter than $M_{V}=-6.5$ should be complete out to $300 \mathrm{kpc}$, and all objects brighter than $M_{V}=-5$ would be detected with at least $50 \%$ efficiency out to this distance.

5. Given the present data, the spatial anisotropy of the MW satellites within DR6 can neither be confirmed nor ruled out.

We provide several different parameterizations of our detection limits to facilitate comparisons between the known MW dwarf galaxy population and predictions. We provide software that returns the detection efficiency of a dwarf galaxy as a function of its luminosity, scale size, distance, and latitude. There are two different codes provided for this; one is based on an analytic description of our detection limits and the other provides a direct interpolation from our 3,825,000 simulated galaxies. We also provide a linear fit as a function of $M_{V}$ of the distance out to which dwarfs are detected with each of $50 \%, 90 \%$, and $99 \%$ efficiency. These fits assume an underlying dwarf galaxy population with combinations of sizes and luminosities similar to those known.

The Southern Sky Survey with the ANU SkyMapper telescope begins in 2009 , which will bring about a way to uniformly search a further $\sim 20,000 \mathrm{deg}^{2}$ of sky for new MW dwarfs. We can naively expect to find around 25 new MW satellites. With a detailed, systematic search covering around three quarters of the sky, we will for the first time be able to conclusively compare the MW satellite galaxy population with theoretical predictions. The apparent anisotropy of the satellites will be conclusively confirmed or ruled out and we will continue to discover the most DM-dominated stellar systems that nature produced. We may also implement improvements to the algorithm to optimize for stellar streams or young stellar populations. McConnachie et al. (2008) have shown that M31 satellites occupy as yet unexplored size-luminosity space around the MW. Surveys beyond SDSS and SkyMapper, such as Pan-STARRS and LSST, will be needed to carefully search for such systems. Even with our carefully characterized detection limits, the true number of MW satellites remains highly uncertain.

Thanks to the anonymous referee, Tony Martin-Jones, Patrick Tisserand, Pavel Kroupa, Iskren Georgiev, Jose Robles, and Charley Lineweaver for helpful comments and discussion. We thank Bill Wyatt and the Telescope Data Center at SAO for maintaining a copy of SDSS at the Harvard-Smithsonian Center for Astrophysics. S.W. thanks the Institute for Theory $\&$ Computation (ITC) at Harvard for support and hospitality and the Smithsonian Astrophysical Observatory for financial support during the final stages of this work. H.J. and S.W. further acknowledge financial support from the Go8/DAAD Australia Germany Joint Research Cooperative Scheme and through the Australian Research Council Discovery Project Grant DP0451426.

\section{REFERENCES}

Adelman-McCarthy, J. K., et al. 2007, ApJS, 172, 634

Adelman-McCarthy, J. K., et al. 2008, ApJS, 175, 297

Bailin, J., Power, C., Norberg, P., Zaritsky, D., \& Gibson, B. K. 2008, MNRAS, 390, 1133

Belokurov, V., et al. 2006a, ApJ, 642, L137

Belokurov, V., et al. 2006b, ApJ, 647, L111

Belokurov, V., et al. 2007, ApJ, 654, 897

Belokurov, V., et al. 2008, ApJ, 686, L83

Brainerd, T. G. 2005, ApJ, 628, L101

Bullock, J. S., Kravtsov, A. V., \& Weinberg, D. H. 2000, ApJ, 539, 517 
Bullock, J. S., Kravtsov, A. V., \& Weinberg, D. H. 2001, ApJ, 548, 33 de Jong, J. T. A., et al. 2008, ApJ, 680, 1112

Dekel, A., \& Silk, J. 1986, ApJ, 303, 39

Diemand, J., Kuhlen, M., \& Madau, P. 2007, ApJ, 667, 859

Fukugita, M., Ichikawa, T., Gunn, J. E., Doi, M., Shimasaku, K., \& Schneider, D. P. 1996 , AJ, 111, 1748

Girardi, L., Grebel, E. K., Odenkirchen, M., \& Chiosi, C. 2004, A\&A, 422, 205

González, R. E., Lares, M., Lambas, D. G., \& Valotto, C. 2006, A\&A, 445, 51

Gunn, J. E., et al. 1998, AJ, 116, 3040

Hogg, D. W., Finkbeiner, D. P., Schlegel, D. J., \& Gunn, J. E. 2001, AJ, 122, 2129

Holmberg, E. 1969, Arkiv for Astronomi, 5, 305

Holtzman, J. A., Afonso, C., \& Dolphin, A. 2006, ApJS, 166, 534

Irwin, M. J. 1994, in Dwarf Galaxies, ed. G. Meylan \& P. Prugniel (Garching: ESO), 27

Irwin, M. J., et al. 2007, ApJ, 656, L13

Ivezić, Ž., et al. 2004, Astron. Nach., 325, 583

Kang, X., Mao, S., Gao, L., \& Jing, Y. P. 2005, A\&A, 437, 383

Keller, S. C., et al. 2007, PASA, 24, 1

Kleyna, J. T., Geller, M. J., Kenyon, S. J., \& Kurtz, M. J. 1997, AJ, 113, 624

Klypin, A., Kravtsov, A. V., Valenzuela, O., \& Prada, F. 1999, ApJ, 522, 82

Klypin, A., Zhao, H., \& Somerville, R. S. 2002, ApJ, 573, 597

Koposov, S., et al. 2007, ApJ, 669, 337

Koposov, S., et al. 2008, ApJ, 686, 279

Kravtsov, A. V., Gnedin, O. Y., \& Klypin, A. A. 2004, ApJ, 609, 482

Kroupa, P., Theis, C., \& Boily, C. M. 2005, A\&A, 431, 517

Liu, C., Hu, J., Newberg, H., \& Zhao, Y. 2008, A\&A, 477, 139

Martin, N. F., de Jong, J. T. A., \& Rix, H.-W. 2008, ApJ, 684, 1075

Martin, N. F., Ibata, R. A., Chapman, S. C., Irwin, M., \& Lewis, G. F. 2007, MNRAS, 380, 281

Mateo, M. L. 1998, ARA\&A, 36, 435

McConnachie, A., et al. 2008, ApJ, 688, 1009

Metz, M., Kroupa, P., \& Jerjen, H. 2007, MNRAS, 374, 1125
Metz, M., Kroupa, P., \& Libeskind, N. I. 2008, ApJ, 680, 287

Moore, B., Ghigna, S., Governato, F., Lake, G., Quinn, T., Stadel, J., \& Tozzi, P. 1999, ApJ, 524, L19

Mori, M., Ferrara, A., \& Madau, P. 2002, ApJ, 571, 40

Okazaki, T., \& Taniguchi, Y. 2000, ApJ, 543, 149

Piatek, S., Pryor, C., Bristow, P., Olszewski, E. W., Harris, H. C., Mateo, M., Minniti, D., \& Tinney, C. G. 2007, AJ, 133, 818

Pier, J. R., Munn, J. A., Hindsley, R. B., Hennessy, G. S., Kent, S. M., Lupton, R. H., \& Ivezić, Ž. 2003, AJ, 125, 1559

Schlegel, D. J., Finkbeiner, D. P., \& Davis, M. 1998, ApJ, 500, 525

Simon, J. D., \& Geha, M. 2007, ApJ, 670, 313

Skrutskie, M. F., et al. 2006, AJ, 131, 1163

Smith, J. A., et al., 2002, AJ, 123, 2121

Somerville, R. S. 2002, ApJ, 572, L23

Strigari, L. E., Bullock, J. S., Kaplinghat, M., Diemand, J., Kuhlen, M., \& Madau, P. 2007, ApJ, 669, 676

Tollerud, E., Bullock, J., Strigari, L., \& Willman, B. 2008, ApJ, 688, 277

Tucker, D. L., et al. 2006, Astronomische Nachrichten, 327, 821

Walsh, S. M., Jerjen, H., \& Willman, B. 2007a, ApJ, 662, L83

Walsh, S. M., Willman, B., Sand, D., Harris, J., Seth, A., Zaritsky, D., \& Jerjen, H. 2007b, ApJ, 688, 245

Whiting, A. B., Hau, G. K. T., Irwin, M., \& Verdugo, M. 2007, AJ, 133, 715

Willman, B. 2003, PhD thesis, Univ. of Washington

Willman, B., Dalcanton, J., Ivezić, Ž., Jackson, T., Lupton, R., Brinkmann, J., Hennessy, G., \& Hindsley, R. 2002, AJ, 123, 848

Willman, B., Governato, F., Dalcanton, J. J., Reed, D., \& Quinn, T. 2004, MNRAS, 353, 639

Willman, B., et al. 2005a, AJ, 129, 2692

Willman, B., et al. 2005b, ApJ, 626, L85

York, D. G., et al. 2000, AJ, 120, 1579

Zentner, A. R., Kravtsov, A. V., Gnedin, O. Y., \& Klypin, A. A. 2005, ApJ, 629, 219

Zucker, D. B., et al. 2006a, ApJ, 643, L103

Zucker, D. B., et al. 2006b, ApJ, 650, L41 